\title{
Financial Entanglement: A Theory of Incomplete Integration, Leverage, Crashes, and Contagion*
}

\author{
Nicolae Gârleanu \\ UC Berkeley-Haas, NBER, and CEPR \\ Stavros Panageas \\ University of Chicago, Booth School of Business and NBER \\ Jianfeng $\mathrm{Yu}$ \\ University of Minnesota, Carlson School of Business
}

May 2014

\begin{abstract}
We propose a unified model of limited market integration, asset-price determination, leveraging, and contagion. Investors and firms are located on a circle, and access to markets involves participation costs that increase with distance. Despite the exante symmetry of investors, their strategies may (endogenously) exhibit diversity, with some investors in each location following high-leverage, high-participation, and highcost strategies and some unleveraged, low-participation, and low-cost strategies. The capital allocated to high-leverage strategies may be vulnerable even to small changes in market-access costs, which can lead to discontinuous price drops, de-leveraging, and portfolio-flow reversals. Moreover, the market is subject to contagion, in that an adverse shock to investors at a subset of locations affects prices everywhere.
\end{abstract}

Keywords: Financial frictions, Market fragmentation, Leverage, Crashes, Contagion JEL Classification: G01, G12

${ }^{*}$ We are grateful for comments and suggestions from Saki Bigio, Xavier Gabaix, Douglas Gale, Michael Gallmeyer, Anna Pavlova, Alp Simsek, and as well as participants at the NBER AP, NBER SI Macro-Finance (ME), Minnesota Macro-AP conference, Studienzentrum Gerzensee, the Cowles Foundation GE conference, LBS Four nations cup, and the Finance Theory Group conferences, and seminar audiences at Univ. of Alberta, Univ. of Birmingham, Columbia Business School, Erasmus University, Hanken, HEC Paris, Univ. of Hong Kong, HKUST, INSEAD, LBS, LSE, Stanford-GSB, Tilburg, Vanderbilt, Univ. of Washington, Yale SOM. 


\section{Introduction}

There is a substantial body of empirical literature (described in detail in the next section) concluding that market integration is limited and risk sharing is imperfect. In particular, a) capital tends to stay "close" to its origin, b) reward for risk reflects - at least partially - "local" factors that one would expect to be diversifiable, and c) reductions in capital flows and the extent of market integration tend to be associated with abrupt and substantial increases in risk premia and drops in prices, along with sudden drops in leverage ("financial crises").

Motivated by these findings, we propose a tractable theoretical framework that offers a unified view of asset-price determination, endogenous market fragmentation, contagion across seemingly unrelated markets, diversity of observed investment strategies, and leverage and its implications for financial crashes.

The model features a continuum of investors and financial markets located on a circle. Investors are endowed with shares of a risky firm domiciled at their location and traded at the same location. The dividends of this security are closely correlated with dividends of securities located in nearby locations and less correlated with securities in distant locations. Even though securities in more distant locations offer greater benefits from the perspective of diversification and risk allocation, participation in such markets involves costs that grow with the distance from the current location. Such costs are reflective of the fact that informational frictions are likely to grow as investors participate in progressively more unfamiliar markets; indeed, inside our model distance on the circle should be viewed as a broad measure encompassing the magnitude of these frictions rather than a narrow measure of geographical distance. Alongside the risky markets, investors have access to a zero-net-supply bond market.

Because access to financial markets is subject to frictions, the market equilibrium features limited integration. Investors from nearby locations, who face similar cost structures, choose to participate on arcs of the circle that feature a high degree of overlap. Even though there may be many assets with no common investor, this overlap renders all markets (indirectly) "entangled."

In such a framework, we examine four sets of issues: a) the determination of asset prices in light of limited market integration; b) the structure of optimal investment strategies, i.e., arcs of participation, leverage, and risky-asset positions chosen by investors; c) the effect of shocks to the financial technology (i.e., the magnitude of market-access costs) and their role in market crashes; and d) the propagation of "local" shocks to the financial technology. We summarize our findings about each of the four issues in turn.

a) Limited market integration implies that investors are over-exposed to the risks of locations in their vicinity. Consequently, risk premia are higher than they would be in a frictionless world. An important aspect of the analysis is that the magnitudes of risk premia and of portfolio flows are tightly linked and reflect the extent of market integration. 
b) A surprising implication of the model is that, although investors in any given location are identical in every respect, their investment strategies may be diverse (that is, feature different participation arcs, risky portfolios, and, importantly, leverage). Specifically, we show that a symmetric equilibrium, i.e., an equilibrium in which investors choose the same strategies may fail to exist. Instead, a fraction of investors chooses strategies featuring high leverage, high Sharpe ratios, and high participation fees, while another fraction of investors chooses unleveraged, low-Sharpe ratio, and low-fee strategies. Prices are determined so that investors are indifferent between the two types of strategies; however, the amount of capital directed to each type of investment strategy is determinate and dictated by market clearing.

The intuition for this finding is that leverage and participation decisions are complements. For a given level of risk premia, participating in more markets increases the maximum Sharpe ratio attainable, which induces investors to take more leverage. Since leverage increases the overall variance of the portfolio, it increases the marginal benefit of further market participation and diversification. This complementarity between participation and leverage decisions gives rise to multiple local extrema in the problem of determining the participation arc. It is noteworthy that these multiple local extrema arise even when the participation costs are convex.

This implication of the model is consistent with the diversity of investment strategies offered to investors by, say, mutual funds and hedge funds. More importantly, it shows that portfolio leverage may arise endogenously in fragmented markets, without requiring inherent differences between investors.

c) An interesting situation emerges when the ability to leverage is not limitless, but rather is subject to a margin constraint. ${ }^{1}$ In such a situation marginal variations in participation costs may cause abrupt changes in equilibrium quantities, and even a change in the type of equilibrium, accompanied by discontinuous drops in prices, leverage, and capital flows.

The intuition is as follows. An increase in participation costs makes investors reluctant to continue allocating funds to the strategies that rely more heavily on costly participation. The resulting outflows from these leveraged strategies reduce aggregate market integration and push down the prices of risky securities. The drop in prices has two effects. On the one hand, it raises the Sharpe ratio and helps restore the attractiveness of high-leverage, broadparticipation strategies. On the other, via the margin constraint, it reduces the possibility of leverage and broad participation because the collateral value of risky securities declines. By constraining leverage below the optimal amount, this second effect necessitates a price drop larger than would obtain in the absence of the margin constraint. Furthermore, when the effect becomes sufficiently strong, further drops in prices can no longer attract enough capital for high-leverage, broad-participation strategies to overcome the simultaneous tightening of the leverage constraint. At this point, a marginal increase in participation costs destroys the pre-existing asymmetric equilibrium and triggers a transition to a new type of equilibrium,

\footnotetext{
${ }^{1}$ In Appendix D we consider alternative formulations of borrowing frictions, motivated by limited liability.
} 
featuring no leveraged strategies. The consequence of this transition is that prices of risky securities, investment across locations, and the amount of leverage in the economy drop discontinuously, despite the smooth dependence of investors' objective functions and feasible choices on participation costs.

d) The fact that markets are only partially integrated may strengthen the interdependence of the prices of risky securities in different locations. We present an example where the financial technology "breaks down" (i.e., the participation costs rise to infinity) only in a subset of locations — we refer to them as the "affected" locations. We show that such an event pushes prices in almost all locations downward. This is true even for locations that are not connected with the affected locations through asset trade and also have negative dividend correlation with them.

The intuition for this finding is as follows. As a direct effect of the shock in the affected regions, investors in affected locations stop investing in risky markets in their vicinity. Consequently, prices in these locations fall so as to attract demand from neighboring unaffected locations. The portfolio reallocation in these neighboring locations happens away from other, farther locations, resulting in weaker demand in these farther locations, necessitating price drops in these locations as well, so as to attract demand from locations neighboring the neighboring locations, etc.

The extent of price drops required to compensate investors for tilting their portfolios to absorb local risks depends on the extent of their overall participation in risky markets. If the extent of their participation is small, so that their portfolio is heavily exposed to risks in their vicinity, then a tilt towards a nearby location requires a higher compensation. By contrast, if investors' portfolios are invested across a broad range of locations, then they are more willing to absorb risks in their vicinity. Hence, somewhat surprisingly, the higher the degree of market integration, the lower the price drop in response to a local participation shock (and vice-versa). ${ }^{2}$

In summary, the paper offers a unified framework to study several financial-market phenomena - such as leveraging, crashes, and contagion — as a result of the interplay between equilibrium prices and (endogenous) market segmentation. Besides the applications that we consider, we believe that the particularly tractable nature of the framework could be useful for studying the numerous instances in economics where frictions prevent optimal risk diversification and capital allocation across asset classes.

This article is related to several strands of theoretical and empirical literature. We discuss connections to the theoretical literature here and postpone a discussion of related empirical literature for the next section.

Through its focus on incomplete participation by all agents, our paper is related to the seminal contribution of Merton (1987) and the studies inspired by it. We endogenize the

\footnotetext{
${ }^{2}$ Interestingly, albeit in a completely different setting, Allen and Gale (2000) also finds that contagion is more severe when financial interconnectivity — between deposit-taking institutions, in that model — is low.
} 
degree of participation, and choose a specific asset-universe structure. Circular structures have been repeatedly used in a variety of applications from location models in industrial organization (Salop (1979)) to the literature on financial networks. ${ }^{3}$ From a technical perspective, our paper proposes a novel and quite tractable structure to model risky payoffs continuously distributed on a circle. The paper also differs from the financial-network literature in setup and questions addressed. Specifically, we study a relatively standard Walrasian exchange economy with (endogenously) limited participation, while that literature typically concentrates on banking issues, network externalities, etc. Finally, our construction of an asymmetric equilibrium is based on Aumann (1966), whose analysis covers the case of nonconcave investor-optimization problems in markets with a continuum of agents.

Price crashes in our model are driven by the interaction of a collateral constraint and a non-concavity in investors' optimization problems, which leads to the adoption of diverse strategies. There exists a vast literature analyzing the interaction between declining prices and tightening collateral constraints - we do not attempt to summarize this literature and simply refer to Kiyotaki and Moore (1997) for a seminal contribution. A distinguishing feature of our analysis is the following tension between two effects of price adjustments. On the one hand, as is common in papers with margin constraints, prices affect feasible leverage choices, thus restricting the set of possible trades. On the other hand, in an equilibrium featuring diverse strategies prices need to adjust to keep investors indifferent between strategies associated with different levels of leverage. This tension is most vividly illustrated in cases of discontinuous equilibrium transitions (crashes), that is, in situations in which no price adjustment can simultaneously satisfy the margin constraint and retain the attractiveness of high-leverage, high-participation strategies. ${ }^{4}$

Modeling price crashes through changes in the type of equilibrium is not new to the literature, which contains a number of papers featuring a combination of backward-bending demand curves and multiple equilibria (see, e.g., Gennotte and Leland (1990), Barlevy and Veronesi (2003), Yuan (2005), and Brunnermeier and Pedersen (2009)). One of our main innovations relative to this literature is the endogenous coexistence of leveraged and unleveraged strategies, which owes to the complementarity we identify between participation and leverage. We also do not rely on asymmetric information and the resulting need to infer from prices, as many of these papers do.

Finally, the domino effect produced by our model relates it to the vast literature on contagion. We do not attempt to summarize this literature. Instead, we single out as a

\footnotetext{
${ }^{3} \mathrm{An}$ indicative and incomplete listing of papers studying networks, and in particular circular ones, in finance includes Allen and Gale (2000), Freixas et al. (2000), Allen et al. (2012), Caballero and Simsek (2012), Acemoglu et al. (2013), and Zawadowski (2013). Allen and Babus (2009) offer a survey of the literature.

${ }^{4}$ Krishnamurthy (2010) discusses two important aspects of a financial crisis: balance-sheet effects and the disengagement of investors from markets (specifically, due to an increase in Knightian uncertainty). Balance-sheet effects and declines in participation are also present in our framework, where the feasibility and the attractiveness of alternative leverage-participation combinations are jointly determined.
} 
natural counterpoint a particular mechanism proposed in many models as an explanation of contagion. Specifically, many papers ${ }^{5}$ posit the existence of some agents, frequently of limited risk-bearing capacity, who price all the assets and whose marginal utility therefore transmits shocks from one asset to the price of another. In contrast, contagion in our model obtains even though no agent participates in all markets. A shock in one area is transmitted to a distant one by affecting first the immediately neighboring areas, which in turn affect their neighboring areas, etc. A practical implication is that there can be positive interdependence between the prices in locations with negatively correlated dividends and no common traders.

The paper is organized as follows. Section 2 presents in greater detail the empirical evidence underlying and motivating this paper. Section 3 presents the baseline model. Section 4 presents the solution and the results. Sections 5 and 6 study crashes, respectively contagion. Finally, extensions, proofs and further discussion on some of the model assumptions are contained in the Appendix.

\section{Motivating Facts}

As a motivation for the assumptions of the model, we summarize some well-documented facts concerning the allocation of capital. Table 1 summarizes the evidence on so-called "gravity" equations in international finance. These gravity equations typically specify a loglinear relation between bilateral flows in various forms of asset trade (equities, bonds, foreign direct investment, etc.) and the sizes of the countries and the geographical distance between them.

A striking and robust finding of this literature is that bilateral capital flows and stocks decay substantially with geographical distance. This finding is surprising, since countries that are geographically distant would seem to offer greater diversification benefits; hence one would expect distance to have the opposite sign from the one found in regressions. The literature typically interprets this surprising finding as evidentiary of informational asymmetries that increase with geographical distance - a crude proxy for familiarity and similarity in social, political, legal, cultural, and economic structures. Supportive of this interpretation is the literature that finds a similar relation between distance and portfolio allocations in domestic portfolio allocations. ${ }^{6}$

\footnotetext{
${ }^{5}$ See, e.g., Kyle and Xiong (2001), Cochrane et al. (2008), or Pavlova and Rigobon (2008).

${ }^{6}$ For instance, Coval and Moskowitz (1999) shows that US mutual fund managers tend to overweight locally headquartered firms. Coval and Moskowitz (2001) shows that mutual fund managers earn higher abnormal returns in nearby investments, suggesting an informational advantage of local investors. Using Finnish data, Grinblatt and Keloharju (2001) shows that investors are more likely to hold and trade the stocks of Finnish firms that are located close to the investors. Similar evidence is presented in Chan et al. (2005): Using mutual fund data from 26 countries, and using distance as a proxy for familiarity, this paper finds that a version of the gravity equation holds for mutual fund holdings. That is, the bias against foreign stocks is stronger when the foreign country is more distant. In a similar vein, Huberman (2001) documents familiarity-related biases in portfolio holdings.
} 
Table 1: Gravity Equations in International Trade and Finance.

The table reports a survey of the literature on gravity equations in international trade and finance. Portes and Rey (2005) uses bilateral equity flow data among 14 countries from 1989 to 1996. Buch (2005) uses the stock of assets and liabilities of banks from 1983 to 1999. Head and Ries (2008) uses bilateral FDI stocks with 30 OECD reporters and 32 partners in year 2001. Talamo (2007) uses FDI flow data from 1980-2001. Aviat and Coeurdacier (2007) uses bilateral trade data and bank asset holding data in year 2001. Ahrend and Schwellnus (2012) uses IMF's Consolidated Portfolio Investment Survey (CPIS) in 2005-2006, which reports bilateral debt investment for 74 reporting countries and 231 partner countries. Typically, the regression performed is

$$
\log \left(X_{i, j}\right)=\alpha+\beta_{1} \log \left(G D P_{i}\right)+\beta_{2} \log \left(G D P_{j}\right)+\beta_{3} \log \left(\text { Distance }_{i, j}\right)+\text { controls }+\epsilon_{i, j}
$$

where $X_{i, j}$ is the equity flow, portfolio holding, FDI flow, FDI stock, trade, or bank asset holdings.

\begin{tabular}{llrr}
\hline Source & Dependent variable & Distance & t-stat \\
\hline Portes and Rey (2005) & Equity flows & -0.881 & -28.419 \\
Buch (2005) & Bank asset holdings & -0.650 & -12.020 \\
Talamo (2007) & FDI flow & -0.643 & -9.319 \\
Head and Ries (2008) & FDI stock & -1.250 & -17.361 \\
Aviat and Coeurdacier (2007) & Trade & -0.750 & -10.000 \\
Aviat and Coeurdacier (2007) & Bank asset holdings & -0.756 & -8.043 \\
Ahrend and Schwellnus (2012) & Bond holdings & -0.513 & -4.886 \\
\hline
\end{tabular}

Further supportive evidence is provided by literature that documents how partial market integration affects the pricing of securities. Bekaert and Harvey (1995) finds that local factors affect the pricing of securities and are not driven out by global factors. An important additional finding of Bekaert and Harvey (1995) is that the relative importance of global and local factors is time varying, suggesting time-varying integration between markets.

Indeed, crisis periods offer an opportunity to visualize the extent of variation in market integration. The left plot of Figure 1 reports the sum of global net purchases of foreign assets by residents (labeled "Gross capital inflows") and the sum of global net purchases of domestic assets by foreigners (labeled "Gross capital outflows"). The figure also reports cross-border bank inflows and outflows based on BIS data. The picture helps visualize that, in the years preceding the financial crisis of 2008, there was a large increase in gross capital flows. This expansion in capital flows came to a sudden stop in the first quarter of 2008, as the financial crisis took hold. In line with the patterns illustrated in the left plot of the figure, Ahrend and Schwellnus (2012) documents a significantly stronger coefficient on distance in cross-border gravity equations during 2008-2009. This evidence suggests that capital becomes more concentrated "locally" during times of crisis.

The right plot of Figure 1 provides a further illustration of capital concentration during the crisis by focusing on readily available BIS data. It depicts the cumulative percentage change in banks' cross-border claims against the respective cumulative change in their lo- 

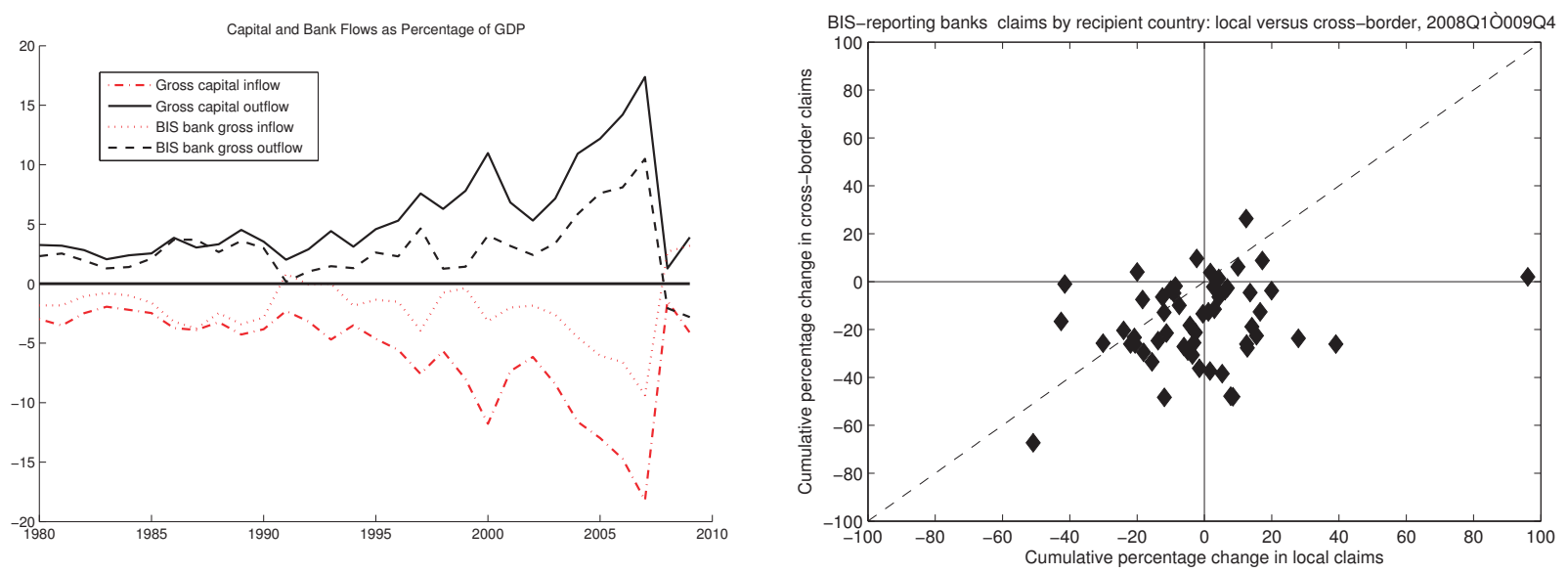

Figure 1: Reversals in global capital flows. Source: Hoggarth et al. (2010). The left plot depicts the sum of global net purchases of foreign assets by residents (labeled "Gross capital inflows") and the sum of global net purchases of domestic assets by foreigners (labeled "Gross capital outflows"). The figure also reports cross-border bank inflows and outflows based on BIS data. Right plot: Cumulative percentage in local claims held by banks against cumulative percentage change in cross-border claims during the 2008Q1 - 2009Q4 period. Source: Hoggarth et al. (2010). based on BIS Data on the 50 largest debtor countries by foreign liabilities. For a detailed description of the sample of reporting countries, see Hoggarth et al. (2010).

cal claims for various countries between 2008Q1 - 2009Q4. Most points on the graph are below the 45-degree line, suggesting that the liquidation of the foreign holdings of banks was disproportionately larger than the respective liquidation of their local holdings. Direct evidence of a "flight to home" effect is provided by Giannetti and Laeven (2012), which shows that the home bias of lenders' loan origination increases by approximately $20 \%$ if the bank's home country experiences a banking crisis. Giannetti and Laeven (2012) also argues that this flight to home effect is distinct from flight to quality, since borrowers of different quality are equally affected.

We note in passing that the increased portfolio concentration during times of crisis is not limited to international data. For instance, the collapse of the (non-agency) securitization market during the financial crisis implied increased limitations to the ability of local issuers to diversify local real-estate risk.

In summary, the empirical evidence supports the following broad conclusions: a) Capital stays "close" to its origin, which implies a "local" concentration of risk in investors' portfolios. b) The extent of market integration is time-varying. Furthermore crises are times when financial integration diminishes quite abruptly. 


\section{Model}

\subsection{Investors and firms}

Time is discrete and there are two dates, $t=0$ and $t=1$. All trading takes place at time $t=0$, while at $t=1$ all payments are made and contracts are settled. Investors are price takers, located at different points on a circle with circumference normalized to one. We index these locations by $i \in[0,1)$. Investors have exponential utilities and maximize expected utility of time-1 wealth

$$
E\left[U\left(W_{1, i}\right)\right]=-E\left[e^{-\gamma W_{1, i}}\right]
$$

where $W_{1, i}$ is the time- 1 wealth of an investor in location $i$. The assumptions that investors only care about terminal wealth and have exponential utility are made for tractability and in order to expedite the presentation of the results. In Appendix A.1 we extend the model to allow for consumption over an infinite horizon.

Besides having identical preferences, investors at any given location are also identical in terms of their endowments and their information sets. Specifically, at time $t=0$ the investors in location $i$ are equally endowed with the total supply of shares (normalized to one) of a competitive, representative firm, which is domiciled at the same location $i$. Each firm pays a stochastic dividend equal to $D_{i}$ in period 1 .

We specify the joint distribution of the dividends $D_{i}$ for $i \in[0,1)$ so as to obtain several properties. Specifically, we wish that 1) firms be ex-ante symmetric, that is, the marginal distribution of $D_{i}$ be independent of $i$;) the total dividends paid $\int_{0}^{1} D_{i} d i$ be constant (normalized to one) ${ }^{7} 3$ ) firms with indices close to each other (in terms of their shortest distance on the circle) experience a higher dividend correlation than firms farther apart; and 4) dividends at different locations be normally distributed.

To formalize these notions, we let $Z_{i}$ denote a standard Brownian motion for $i \in[0,1]$, and we also introduce the quantity

$$
B_{i} \equiv Z_{i}-i Z_{1} \text { for } i \in[0,1]
$$

known as a Brownian bridge. Two immediate implications of the construction (2) are that the Brownian bridge satisfies $B_{0}=B_{1}=0$ and has continuous paths (a.s.). ${ }^{8}$ Using the

\footnotetext{
${ }^{7}$ This assumption is made purely for ease of exposition. It is straightforward to introduce aggregate risk by adding a common, normally distributed disturbance to dividends in all locations, without affecting our results.

${ }^{8}$ Equivalently, a Brownian bridge $B_{t}$ on $[0,1]$ is a process distributed as the Brownian motion $Z_{t}$ conditional on $Z_{1}=0$.
} 

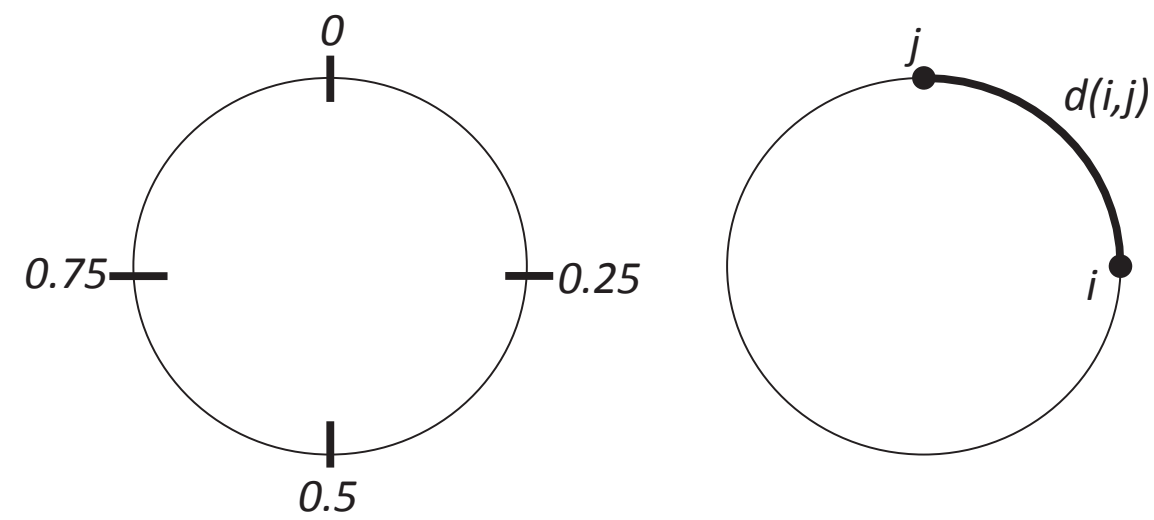

Figure 2: The left plot depicts a circle with circumference 1. The bold arc on the right plot depicts the notion of the shortest distance on the circle $d(i, j)$ between points $i$ and $j$ that we use throughout.

definition of $B_{i}$, we let $D_{i}$ be defined as

$$
D_{i} \equiv 1+\sigma\left(B_{i}-\int_{0}^{1} B_{j} d j\right)
$$

where $\sigma>0$ is a constant controlling the volatility of the dividend process. Since the specification (3) plays a central role in our analysis, we first derive the statistical properties of $D_{i}$, and then we provide a graphical illustration.

Lemma $1 D_{i}$ satisfies the following properties.

1. (Symmetry and univariate normality) The marginal distribution of $D_{i}$ is the same for all $i$. Specifically, $D_{i}$ is normally distributed with mean 1 and variance $\frac{\sigma^{2}}{12}$.

2. (No aggregate risk) $\int_{0}^{1} D_{i} d i=1$.

3. (Continuity on the circle) Let $d(i, j) \equiv \min (|i-j|, 1-|i-j|)$ denote a metric on the interval $[0,1)$. Then $D_{i}$ is continuous (a.s.) on $[0,1)$ if the interval $[0,1)$ is endowed with the metric $d$.

4. (Joint normality and distance-dependent covariance structure) For any vector of locations $i=\left(i_{1}, i_{2}, \ldots, i_{N}\right)$ in $[0,1)$, the dividends $\left(D_{i_{1}}, \ldots, D_{i_{N}}\right)$ are joint normal, with covariances given by

$$
\operatorname{cov}\left(D_{i_{n}}, D_{i_{k}}\right)=\sigma^{2}\left(\frac{1}{12}-\frac{d\left(i_{n}, i_{k}\right)\left(1-d\left(i_{n}, i_{k}\right)\right)}{2}\right) .
$$

It is easiest to understand the properties of $D_{i}$ by using a graphical illustration. The left plot of Figure 2 provides an illustration of the interval [0,1) "wrapped" around as a circle 

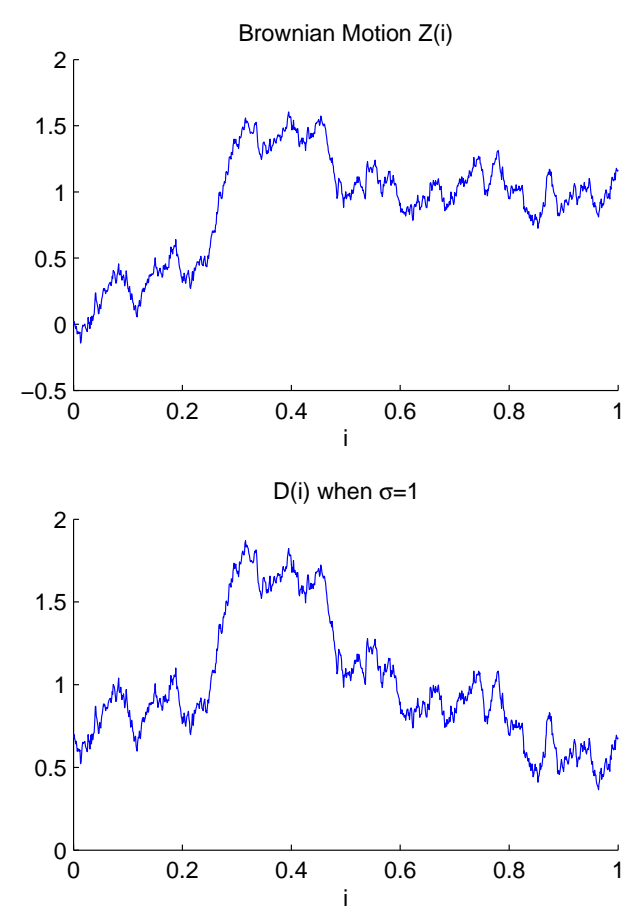

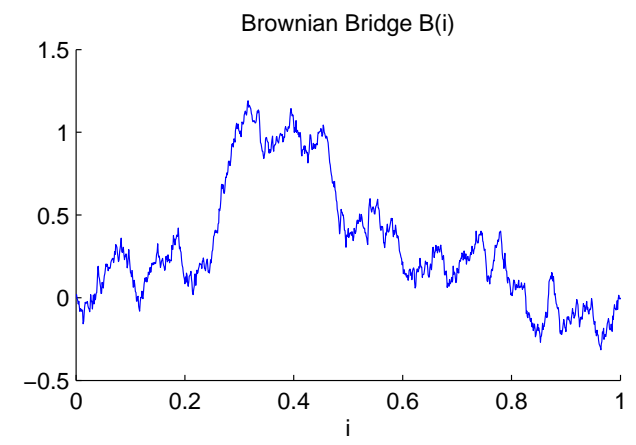

$\mathrm{D}(\mathrm{i})$ (polar coordinates)

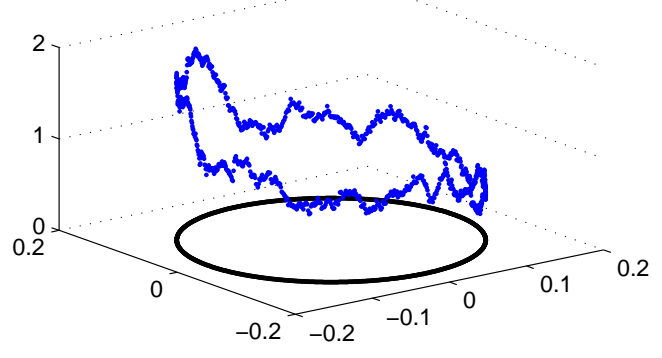

Figure 3: An illustration of the construction of the dividend process $D_{i}$. The two plots at the top depict a sample Brownian path $Z_{i}$, and the associated path of a Brownian bridge $B_{i}$. The two bottom plots depict the associated sample path of $D_{i}$, when the indices $i \in[0,1)$ are aligned on a line and when the same interval is depicted as a circle with circumference one.

with circumference one. The metric $d(i, j)$ can be thought of as the length of the shortest arc connecting $i$ and $j$, as the right plot of Figure 2 illustrates. Figure 3 illustrates a path of $Z_{i}$ and the associated paths of $B_{i}$ and $D_{i}$.

A remarkable property of the dividend structure (3) is that the covariance, and therefore correlation, of dividends in any locations $i$ and $j$ depend exclusively on the distance $d(i, j)$ between the two locations, but not the locations themselves. Equation (4) gives an explicit expression for the covariance of the dividends at different locations. The associated correlations follow immediately as

$$
\operatorname{corr}\left(D_{i}, D_{j}\right)=1-6 d(i, j)(1-d(i, j))
$$

Equation (5) implies that the correlation between $D_{i}$ and $D_{j}$ approaches one as the distance $d(i, j)$ approaches zero, and is minimized when $d(i, j)=\frac{1}{2}$, i.e., when the two firms are located diametrically opposite each other on the circle.

An implication of Lemma 1 (property 2) is that all risk in the economy is diversifiable. It is straightforward to introduce systematic risk by simply adding a common, normally distributed shock to all dividends $D_{i}$. Such a shock would require an additional risk premium, 
but would not alter any of our results otherwise. In the interest of simplicity and to emphasize that our focus is on frictions that assign a positive risk price to diversifiable risk, we choose to not include aggregate risk.

\subsection{Financial markets and participation costs}

Investors in location $i$ can trade claims to the stock of firms located in every location $j \in[0,1)$ on the circle. Motivated by the evidence presented in Section 2 - specifically the fact that capital tends to stay close to its origin — we assume that participation in financial markets is costly, and the more so the farther away a financial market is from an investor's location. In the Appendix $\mathrm{C}$ we provide one possible motivation for such costs, namely as informationacquisition costs. Specifically we present an expanded version of the model with multiple securities of unobserved quality in every location; investors have to incur a distance-dependent cost to overcome informational asymmetries that lower their returns when investing in distant locations. Indeed, the appendix shows that the comparatively higher returns obtained by an investor in locations where she is informed can render uninformed participation suboptimal; alternatively phrased, investors participate only in locations where they choose to pay the cost to become informed. We present the details of this model extension in Appendix C, and proceed with our analysis taking the participation costs as given.

We next propose a mathematical structure for the participation costs. The participation decision of investors consists of choosing a subset of all markets $[0,1)$ in which to invest. To avoid unnecessary complications, we restrict attention to subsets of $[0,1)$ that can be represented as a finite union of disjoint intervals with midpoints $a_{i, n}$ and lengths $\Delta_{i, n}$. Hence, the participation-cost function is a mapping from such subsets of $[0,1)$ to the set of positive real numbers. For instance, if an investor in location $i$ chooses to participate in $N_{i}$ such intervals with midpoints $a_{i, n}$ and total length $\Delta_{i} \equiv \sum_{n=1}^{N_{i}} \Delta_{i, n}$, she incurs costs equal to

$$
F_{i}\left(\left\{a_{i, 1}, \ldots, a_{i, N_{i}}\right\}, \Delta_{i}\right)=\kappa\left(b_{N_{i}} \sum_{n=1}^{N_{i}} f\left(d\left(a_{i, n}, i\right)\right)+g\left(\Delta_{i}\right)\right)
$$

where $\kappa>0$, and $f:\left(0, \frac{1}{2}\right] \rightarrow \mathbb{R}$ and $g:[0,1) \rightarrow \mathbb{R}$ are positive, non-decreasing, differentiable, and convex functions. We assume that $f$ has a discontinuity at zero in the sense that $\lim _{x \rightarrow 0} f(x)>0$, while $f(0)=0$. Also, $g(0)=0$ and $g^{\prime}(0)=0$. Finally, $b_{N_{i}}$ is positive and non-decreasing in $N_{i}$; we set $b_{1}=b_{2}=1$ without loss of generality. ${ }^{9}$

We make several remarks on specification (6). First we note that $F_{i}(\{i\}, 0)=0$, so that participating only in the local market is costless. Second, the fact that $f$ is increasing implies that investing in markets that are farther away (in the sense that the distance $d\left(a_{i, n}, i\right)$ from

\footnotetext{
${ }^{9}$ Since $f(0)=0$, the first mid-point that an investor chooses is always her home location. Hence $b_{1}$ can be chosen arbitrarily, as $b_{1} f(0)=0$. Similarly, $b_{2}=1$ is without loss of generality, since we can always re-define $f^{*}=b_{2} f$ and $b_{N}^{*}=\frac{b_{N}}{b_{2}}$ without changing the total cost $F_{i}$.
} 

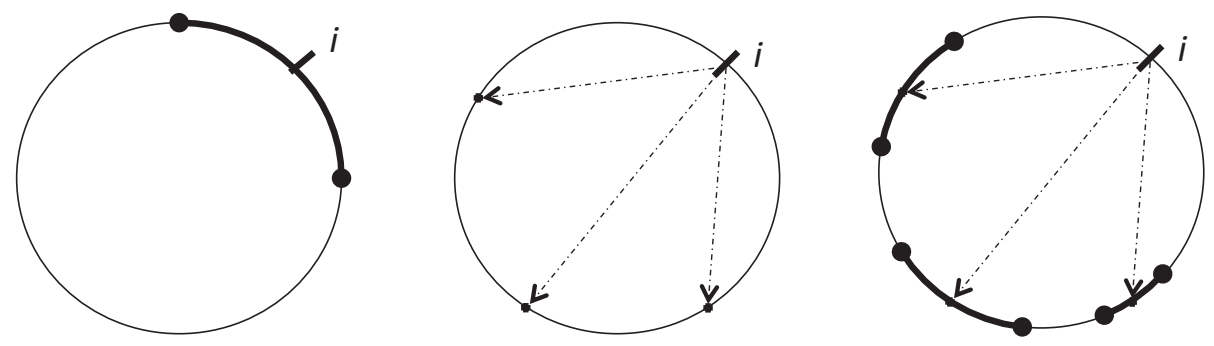

Figure 4: Illustration of participation choices under different assumptions on the participation $\operatorname{costs} F_{i}\left(\left\{a_{i, 1} ; . . ; a_{i, N_{i}}\right\}, \Delta_{i}\right)$.

one's location is larger) is more costly than participating in markets that are close by. Third, increasing the total mass of markets in which the investor participates $\left(\Delta_{i}\right)$, while keeping the number (and midpoints) of intervals the same, incurs incremental rather than fixed costs. This captures the idea that expanding participation to contiguous markets is substantially less costly than participating in a market that is not adjacent to any of the markets where the investor has already decided to participate. Fourth, the fact that participation costs depend on the location of the investor implies that investors in different locations on the circle face different costs of participating in a given market $j$, depending on their proximity to that market. Finally, for technical convenience and ease of exposition, the costs of expanding the total measure of the interval are specified as independent of the structure of the midpoints and the lengths of the subintervals. This assumption is not crucial and can be easily relaxed. ${ }^{10}$

Even though one can specify more elaborate participation-cost structures, the one given by (6) is sufficiently flexible for our purposes. To better understand this cost structure it is useful to consider some special cases. In the first special case, the investor can only participate in an arc centered at her home location (depicted in the left plot of Figure 4). This outcome follows from (6) if one sets $b_{N} f(x)=\infty$ and $g(y)<\infty$ for any $x>0$, $y>0$, and $N>1$, so that the participation cost reduces to $F\left(\Delta_{i}\right)=\kappa g\left(\Delta_{i}\right)$. We note that in this special case the participation-cost structure is convex (unlike when the investor participates in disjoint intervals, which carries fixed costs). This convexity is quite attractive for illustrating some of the results of the paper, and therefore this special case will play an important role in our analysis. A second special case corresponds to situations in which an investor only participates on discrete points (middle plot of Figure 4). This outcome obtains from (6) if $b_{N} f(x)<\infty$ and $g(y)=\infty$ for any $y>0$. In the general case $b_{N} f(x)<\infty$ and $g(y)<\infty$, the participation decision involves choosing the number of points $N_{i}$, the location of the midpoints $a_{i, n}$, and the length of the intervals $\Delta_{i, n}$ at each location. This situation is depicted in the right plot of Figure 4.

Besides the markets for risky shares, there is a market for zero-net-supply riskless bonds

\footnotetext{
${ }^{10}$ Indeed, if one were to replace $g\left(\Delta_{i}\right)$ with $\sum_{n=1}^{N_{i}} g\left(\Delta_{i, n}\right)$, that would introduce additional reasons for the cost structure to be non-convex, strengthening some of the conclusions of the paper.
} 
that pay one unit of wealth at time 1. Participation in the bond market is costless for everyone.

The participation costs act as deadweight costs that are paid out (i.e., reduce consumption) at time 1.

We remark in passing that our results would not be affected if, instead of making the participation decisions themselves, agents invested through frictionless and competitive intermediation. That is, we could introduce a competitive sector of intermediaries who incur the participation costs, choose optimal portfolios for their clients, and then charge them competitive fees to cover the participation costs. ${ }^{11}$

\subsection{Individual maximization and equilibrium}

To formalize an investor's decision problem we let $P_{i}$ denote the price of a share in a market $i, P_{B} \equiv \frac{1}{1+r}$ the price of a bond, $d X_{j}^{(i)}$ the mass of shares in market $j$ bought by an investor located in $i,{ }^{12}$ and $X_{B}^{(i)}$ the respective number of bonds. Then, letting

$$
W_{0, i} \equiv \int_{0}^{1} P_{j} d X_{j}^{(i)}+P_{B} X_{B}^{(i)}
$$

denote the total financial wealth of an investor in location $i$, the budget constraint of an investor can be expressed as $W_{0, i}=P_{i}$.

We are now in a position to formulate the investor's maximization problem as

$$
\max _{w_{i}^{f}, G^{(i)}, N_{i}, \vec{a}_{i}, \vec{\Delta}_{i}} E\left[U\left(W_{1, i}\right)\right]
$$

subject to the budget constraint $W_{0, i}=P_{i}$, and

$$
W_{1, i}=W_{0, i}\left(w_{i}^{f}(1+r)+\left(1-w_{i}^{f}\right) \int_{0}^{1} R_{j} d G_{j}^{(i)}\right)-F_{i},
$$

where $w_{i}^{f}$ is the fraction of $W_{0, i}$ invested in the risk-free security by an agent in location $i$, $G_{j}^{(i)}$ is a bounded-variation function with $\int_{0}^{1} d G_{j}^{(i)}=1$, which is constant in locations where the investor does not participate (i.e., $d G_{j}^{(i)}=0$ in these locations), so that $d G_{j}^{(i)}$ captures the

\footnotetext{
${ }^{11}$ In a previous version of the paper, we consider such a model. Specifically, in this model a) investors don't have direct access to markets, but rather have to hire (competitive) intermediaries in their location to gain access to distant markets, b) the intermediaries attract clients by offering portfolios that maximize their welfare, and c) intermediaries charge clients fees to cover the participation costs that they incur. Not surprisingly, the lack of frictions between investors and intermediaries implies that the allocations and the prices in the two models are identical.

${ }^{12}$ The function $X_{j}^{(i)}$ has finite variation. We adopt the natural convention that $X_{j}^{(i)}$ is continuous from the right and has left limits.
} 
fraction of the risky component of the portfolio $\left(1-w_{i}^{f}\right) W_{0, i}$ invested in the share of stock $j$ by a consumer located in $i$. Finally, $R_{i} \equiv \frac{D_{i}}{P_{i}}$ is the realized gross return on security $i$ at time 1 . We do not restrict $G$ to be continuous, that is, we allow investors to invest mass points of wealth in some locations.

The definition of equilibrium is standard. An equilibrium is a set of prices $P_{i}$, a real interest rate $r$, and participation and portfolio decisions $w_{i}^{f}, G^{(i)}, N_{i}$, and $\left\{a_{i, 1}, \ldots, a_{i, N_{i}}, \Delta_{i, 1}, \ldots, \Delta_{i, N_{i}}\right\}$ for all $i \in[0,1]$ such that: 1) $w_{i}^{f}, G^{(i)}, N_{i}$, and $\left\{a_{i, 1 \ldots} a_{i, N_{i}}, \Delta_{i, 1 \ldots} \Delta_{i, N_{i}}\right\}$ solve the optimization problem of equation $(7), 2)$ financial markets for all stocks clear: $P_{j}=\int_{i \in[0,1]}\left(1-w_{i}^{f}\right) W_{0, i} d G_{j}^{(i)}$, and 3) the bond market clears, i.e., $\int_{i \in[0,1]} W_{0, i} w_{i}^{f} d i=0$.

By Walras' law, we need to normalize the price in one market. Since in the baseline model we abstract from consumption at time zero for parsimony, we normalize the price of the bond to be unity (i.e., we choose $r=0$ ). We discuss consumption at more dates than time one and an endogenously determined interest rate in Appendix A.1.

\section{Solution and Its Properties}

We solve the model and illustrate its properties in a sequence of steps. First, we discuss a frictionless benchmark, where participation costs are absent. Second, in order to highlight the core intuition, we discuss the special case where the investor can only participate in an arc centered at her location, so that her participation decision amounts to choosing the length of that arc (the special case depicted on the left panel of Figure 4). We consider the general case, which allows for both contiguous and non-contiguous participation choices, in Appendix A.2.

\subsection{A frictionless benchmark}

As a benchmark, we consider first the case without participation costs: $F_{i}=0$. In this case, the solution to the model is trivial. Every investor $i$ participates in every market $j$. The first order condition for portfolio choice is

$$
E\left[U^{\prime}\left(W_{1, i}\right)\left(R_{j}-(1+r)\right)\right]=0 .
$$

With the above first-order condition in hand, one can verify the validity of the following (symmetric) equilibrium: $w_{i}^{f}=0$, and $G_{j}^{(i)}=G_{j}=j$ for all $(i, j) \in[0,1) \times[0,1)$ - i.e., investors in all locations $i$ choose an equally weighted portfolio of every share $j \in[0,1]$. Accordingly, $W_{1, i}=\int_{0}^{1} D_{j} d j=1$. Since in this equilibrium $W_{1, i}=1$, the Euler equation (9) implies that

$$
E\left(R_{j}\right)=1+r
$$


Combining (10) with the definition $R_{j}=\frac{D_{j}}{P_{j}}$ implies

$$
P_{j}=\frac{E\left(D_{j}\right)}{1+r}=\frac{1}{1+r}=1
$$

where the last equation follows from the normalization $r=0$.

The equilibrium in the frictionless case is intuitive. Since there are no participation costs, investors hold an equally weighted portfolio across all locations. Since — by assumption there is no risk in the aggregate, the risk of any individual security is not priced.

\subsection{Symmetric equilibria with participation costs}

To facilitate the presentation of some of the key results, we focus on the special case depicted in the left plot of Figure 4, i.e., the case where the investor's participation choice amounts to choosing the length $\Delta$ of an arc centered at her location. The associated participation cost is $\kappa g(\Delta)$.

To make the investor's problem well-defined and interesting, we assume that the costs of participation increase sufficiently fast (and thus become infinite) as $\Delta$ approaches one:

Assumption $1 \lim _{\Delta \rightarrow 1} g^{\prime}(\Delta)(1-\Delta)^{4}=\infty$.

Assumption 1 helps ensure that an optimal $\Delta$ less than one exists for every investor. ${ }^{13}$

Before presenting results, we introduce a convention to simplify notation.

Convention 1 For any real number $x$, let $\lfloor x\rfloor$ denote the floor of $x$, i.e., the largest integer weakly smaller than $x$. We henceforth use the term "location $x$ " (on the circle) to refer to the unique point in $[0,1)$ given by $x \bmod 1 \equiv x-\lfloor x\rfloor$.

With this convention we can map any real number to a unique location on the circle with circumference one. For example, this convention implies that the real numbers $-0.8,0.2$, and 1.2 correspond to the same location on the circle with circumference one, namely 0.2. An implication of this convention is that any function $h$ defined on the circle extends to a function $\hat{h}$ on the real line that is periodic with period one, i.e., $\hat{h}(x)=\hat{h}(x+1)=h(x$ mod 1). From now on, we treat functions on the circle also function defined on the entire real line.

We next introduce two definitions.

\footnotetext{
${ }^{13}$ If $P<1$, so that there is a risk premium, the limiting case $\Delta=1$ is not well defined, since both the costs and the benefits of participation diverge to infinity as $\Delta \rightarrow 1$. Assumption 1 along with an application of L'Hôpital's rule ensures that the cost component dominates the benefit component as $\Delta \rightarrow 1$, resulting in an optimal (interior) choice of $\Delta \in[0,1)$. We also note that, if we introduce aggregate risk, then Assumption 1 can be replaced with the weaker $\lim _{\Delta \rightarrow 1} g(\Delta)=\infty$. Finally, no assumption is necessary in the presence of a leverage constraint, as in Section 5 (except that $g(\Delta)>0$ for some $\Delta \in(0,1)$ ).
} 
Definition 1 The standardized portfolio associated with $G_{j}^{(i)}$ is the function $L: \mathbb{R} \rightarrow[0,1]$ defined by $L_{j}=G_{i+j}^{(i)}$.

The notion of a standardized portfolio allows us to compare portfolios of investors at different locations on the circle. For example, if all investors choose portfolios with weights that only depend on the distance $d$ between their domicile and the location of investment, and these distance-dependent weights are the same for all investors, then these investors hold the same standardized portfolio.

Definition 2 A symmetric equilibrium is an equilibrium in which all agents choose the same participation interval $\Delta$, the same leverage $w^{f}$, and the same standardized portfolio.

Due to the symmetry of the problem, it is natural to start by attempting to construct a symmetric equilibrium.

Proposition 1 For any $\Delta \in(0,1)$ define

$$
L_{j}^{*} \equiv \begin{cases}0 & \text { if } j \in\left[-\frac{1}{2},-\frac{\Delta}{2}\right) \\ j+\frac{1}{2} & \text { if } j \in\left[-\frac{\Delta}{2}, \frac{\Delta}{2}\right) \\ 1 & \text { if } j \in\left[\frac{\Delta}{2}, \frac{1}{2}\right)\end{cases}
$$

and

$$
\omega(\Delta) \equiv \operatorname{Var}\left(\int_{0}^{1} D_{j} d L_{j}^{*}\right)=\frac{\sigma^{2}}{12}(1-\Delta)^{3} .
$$

Finally, let $\Delta^{*}$ denote the (unique) solution to the equation

$$
\kappa g^{\prime}\left(\Delta^{*}\right)=-\frac{\gamma}{2} \omega^{\prime}\left(\Delta^{*}\right)
$$

and also consider the set of prices

$$
P_{i}=P \equiv 1-\gamma \omega\left(\Delta^{*}\right)
$$

Then, assuming that a symmetric equilibrium exists, the choices $\Delta^{(i)}=\Delta^{*}, w_{i}^{f}=0, d G_{j}^{(i)}=$ $d L_{j-i}^{*}$, and prices $P_{i}=P$ constitute the unique symmetric equilibrium.

Proposition 1 gives simple, explicit expressions for both the optimal portfolios and participation intervals. To understand how these quantities are derived, we take an individual agent's wealth $W_{1, i}$ from (8) and we assume that prices for risky assets are the same in all 
locations. Then, using $W_{0, i}=P_{i}=P, r=0, R_{j}=\frac{D_{j}}{P}$, and $F_{i}=\kappa g(\Delta)$, we express $W_{1, i}$ as

$$
\begin{aligned}
W_{1, i} & =P\left(w_{i}^{f}+\left(1-w_{i}^{f}\right) \int_{0}^{1} R_{j} d G_{j}^{(i)}\right)-\kappa g(\Delta) \\
& =\left(P w_{i}^{f}+\left(1-w_{i}^{f}\right) \int_{0}^{1} D_{j} d G_{j}^{(i)}\right)-\kappa g(\Delta) .
\end{aligned}
$$

Because of exponential utilities and normally distributed returns, maximizing $E U\left(W_{1, i}\right)$ is equivalent to solving

$$
\max _{d G_{j}^{(i)}, \Delta, w_{i}^{f}} P w_{i}^{f}+\left(1-w_{i}^{f}\right) \int_{0}^{1} E D_{j} d G_{j}^{(i)}-\frac{\gamma}{2}\left(1-w_{i}^{f}\right)^{2} \operatorname{Var}\left(\int_{0}^{1} D_{j} d G_{j}^{(i)}\right)-\kappa g(\Delta) .
$$

Noting that $E D_{j}=1$, inspection of equation (16) shows that (for any $w^{f}$ and $\Delta$ ) the optimal portfolio is the one that minimizes the variance of dividends in the participation interval $\Delta$. Since the covariance matrix of dividends is location invariant, the standardized varianceminimizing portfolio is the same at all locations. Solving for this variance-minimizing portfolio $L_{j}$ is an infinite-dimensional optimization problem. However, because of the symmetry of the setup we are able to solve it explicitly, and equation (12) provides the solution. The optimal portfolio, $L_{j}^{*}$, corresponds to the distribution that minimizes the sum of the vertical distances to the uniform distribution on $\left[-\frac{1}{2}, \frac{1}{2}\right)$, subject to the constraints that $L_{j}=0$ for $j \in\left[-\frac{1}{2},-\frac{\Delta}{2}\right)$ and $L_{j}=1$ if $j \in\left[\frac{\Delta}{2}, \frac{1}{2}\right)$. The resulting optimized variance is given by $\omega(\Delta)$, defined in (13).

Accordingly, the agent's problem can be written more compactly as

$$
V=\max _{\Delta, w_{i}^{f}} P w_{i}^{f}+\left(1-w_{i}^{f}\right)-\frac{\gamma}{2}\left(1-w_{i}^{f}\right)^{2} \omega(\Delta)-\kappa g(\Delta) .
$$

The first-order conditions with respect to $w_{i}^{f}$, respectively $\Delta$, are

$$
\begin{aligned}
1-P & =\gamma\left(1-w_{i}^{f}\right) \omega(\Delta) \\
\kappa g^{\prime}(\Delta) & =-\frac{\gamma}{2}\left(1-w_{i}^{f}\right)^{2} \omega^{\prime}(\Delta) .
\end{aligned}
$$

Since in a symmetric equilibrium market clearing requires $w_{i}^{f}=0$, equation (18) becomes identical to (15) and (19) becomes equivalent to (14).

Equation (14) makes explicit the resolution of the tradeoff between participation costs and risk taking. Specifically, the participation interval is determined as the point where the marginal cost of participation, $\kappa g^{\prime}(\Delta)$, is equal to the marginal benefit of participation, $-\frac{\gamma}{2} \omega^{\prime}(\Delta)$.

Figure 5 illustrates this tradeoff by plotting the marginal cost from increasing $\Delta$, namely 


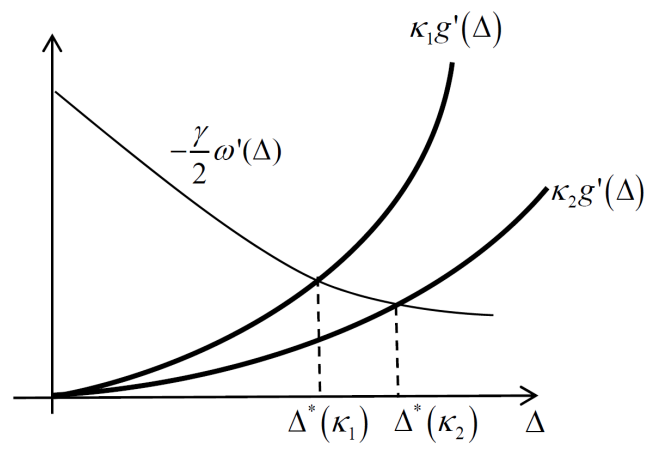

Figure 5: Illustration of the determination of $\Delta^{*}$ when a symmetric equilibrium exists. The figure illustrates that $\Delta^{*}$ increases when $\kappa_{2}<\kappa_{1}$.

$\kappa g^{\prime}(\Delta)$, against the respective marginal benefit $-\frac{\gamma}{2} \omega^{\prime}(\Delta)$. Since $g(\Delta)$ is convex, $g^{\prime}(\Delta)$ is upward sloping. By contrast, the marginal benefit is declining, since $-\omega^{\prime \prime}(\Delta)=-\frac{\sigma^{2}}{2}(1-\Delta)<$ 0 . Since $g^{\prime}(0)=0, \lim _{\Delta \rightarrow 1} g^{\prime}(\Delta)=\infty,-\omega^{\prime}(0)>0$, and $\omega^{\prime}(1)=0$, the two curves intersect at a unique point $\Delta^{*} \in[0,1)$.

Proposition 1 helps capture the economic mechanisms that underlie our model. Consider, for instance, its implications for a reduction in the cost of accessing markets (i.e., a reduction in $\kappa)$. As Figure 5 illustrates, such a reduction increases the degree of participation $\Delta$ and promotes portfolio flows across different locations. In turn, this increased participation improves risk sharing across different locations, which leads to higher prices of risky securities, $P=1-\gamma \omega(\Delta)$, and accordingly lower risk premia. By contrast, an increase in the costs of accessing risky markets leads to a lower $\Delta$ and a higher degree of concentration of risk. The resulting decline in the extent of risk sharing leads to a drop in the prices of risky assets and an increase in risk premia.

These mechanisms of the model capture the stylized facts summarized in Section 2. We highlight one aspect of our analysis: the extent of market integration and cross-location portfolio flows, on one hand, and the magnitude of risk premia, on the other, are intimately linked. By contrast, representative-agent approaches to the determination of risk premia are - by their construction - limited in their ability to explain the empirically prevalent joint movements in risk premia and portfolio movements, since the representative agent always holds the market portfolio and prices adjust so as to keep the agent content with her holdings.

We conclude this section by noting that in Appendix A.1 we extend the results obtained so far to an intertemporal version of the model with recurrent shocks to participation costs. That extension strengthens the comparative statics conclusions established above by showing that, in a dynamic setting with participation-cost shocks, the model produces a) a negative correlation between capital flows and excess returns, b) time variation in excess returns that is unrelated to expected dividend growth, aggregate output etc., and c) return correlation across locations that exceeds the respective correlation of dividends. Furthermore, the additional risk caused by variation in the participation costs is priced, resulting in an increased 
expected excess return.

Next we turn our attention to a central issue of our analysis, pertaining to the existence of a symmetric equilibrium.

\subsection{Asymmetric, location-invariant equilibria: Leverage and the diversity of financial strategies}

Proposition 1 contains the premise that a symmetric equilibrium exists. Surprisingly, despite the symmetry of the model setup, a symmetric equilibrium may fail to exist. Instead, the market equilibrium may involve different choices (leverage ratios, portfolios of risky assets, wealth allocations, etc.) for agents in the same location, even though these agents have the same preferences and endowments and are allowed to make the same participation and portfolio choices.

These claims are explained by the observation that the necessary first-order conditions resulting in the prices $P_{i}=P=1-\gamma \omega\left(\Delta^{*}\right)$ are not generally sufficient. We now take a closer look at whether a symmetric equilibrium exists. Specifically, we fix the price $P=1-\gamma \omega\left(\Delta^{*}\right)$ of Proposition 1 and investigate whether (and under what conditions) the choices $w^{f}=0$ and $\Delta=\Delta^{*}$ are indeed optimal for the investor.

To answer this question, we consider again the maximization problem (17). Taking $P=1-\gamma \omega\left(\Delta^{*}\right)$ as given, substituting into (18), and re-arranging implies that if investors allocate their wealth over a participation interval $\Delta$ (potentially different from $\Delta^{*}$ ), then

$$
1-w^{f}=\frac{\omega\left(\Delta^{*}\right)}{\omega(\Delta)}
$$

Equation (20) contains an intuitive prediction. An investor allocating her wealth over a span $\Delta>\Delta^{*}$ is facing the same average returns, but a lower variance $\omega(\Delta)$, and hence a higher Sharpe ratio compared to an investor allocating her wealth over an interval of size $\Delta^{*}$. Accordingly, the former investor finds it optimal to leverage her portfolio. This is reflected in equation (20), which states that $w^{f}<0\left(w^{f}>0\right)$ whenever $\Delta^{*}<\Delta\left(\Delta^{*}>\Delta\right)$.

An interesting implication of (19) is that the marginal benefit of an increased participation interval becomes larger with leverage. This is intuitive since increased leverage implies a more volatile wealth next period and hence a higher marginal benefit of reducing that variance by increasing $\Delta$. In short, the choices of $\Delta$ and of leverage $1-w^{f}$ are complements.

This complementarity can lead to an upward sloping marginal benefit of increased participation. Indeed, with our cash-flow specification, substituting (20) into (19) gives

$$
\kappa g^{\prime}(\Delta)=-\frac{\gamma}{2}\left(\frac{\omega\left(\Delta^{*}\right)}{\omega(\Delta)}\right)^{2} \omega^{\prime}(\Delta) .
$$



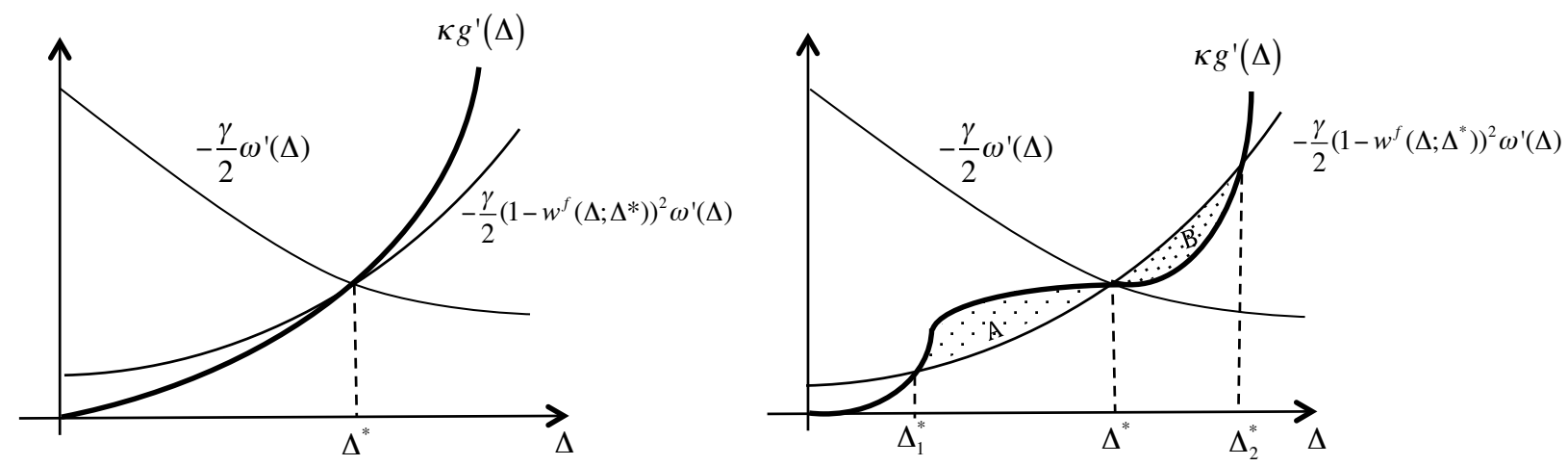

Figure 6: Illustration of the marginal benefit curve $-\frac{\gamma}{2}\left(\frac{\omega\left(\Delta^{*}\right)}{\omega(\Delta)}\right)^{2} \omega^{\prime}(\Delta)$, the marginal cost curve $\kappa g^{\prime}(\Delta)$ and the marginal benefit curve $-\frac{\gamma}{2} \omega^{\prime}(\Delta)$ restricting $w^{f}=0$. The left plot illustrates a case where a symmetric equilibrium exists whereas the right plot illustrates a case where a symmetric equilibrium fails to exist.

Using the fact $\omega(\Delta)=\frac{\sigma^{2}}{12}(1-\Delta)^{3}$, the right hand side can be expressed as $-\frac{\gamma}{2}\left(\frac{\omega\left(\Delta^{*}\right)}{\omega(\Delta)}\right)^{2} \omega^{\prime}(\Delta)=$ $\frac{\gamma \sigma^{2}}{8}\left(1-\Delta^{*}\right)^{6}(1-\Delta)^{-4}$, which is increasing in $\Delta$.

Figure 6 helps illustrate these notions. The figure depicts the marginal cost curve $\kappa g^{\prime}(\Delta)$, the marginal benefit curve $-\frac{\gamma}{2}\left(1-w^{f}\left(\Delta ; \Delta^{*}\right)\right)^{2} \omega^{\prime}(\Delta)=-\frac{\gamma}{2}\left(\frac{\omega\left(\Delta^{*}\right)}{\omega(\Delta)}\right)^{2} \omega^{\prime}(\Delta)$, and also the curve $-\frac{\gamma}{2} \omega^{\prime}(\Delta)$, i.e., the marginal benefit of participation fixing $w^{f}=0$. The point where all three curves intersect corresponds to the point $\Delta=\Delta^{*}$. The left plot of Figure 6 illustrates a case where a symmetric equilibrium exists, whereas the right plot illustrates a case where a symmetric equilibrium fails to exist. The difference between the two plots is the shape of $g^{\prime}(\Delta)$. In the left plot $g^{\prime}(\Delta)$ intersects the marginal benefit curve only once, namely at $\Delta^{*}$. For values smaller than $\Delta^{*}$, the marginal benefit of participation is above the marginal cost and vice versa for values larger than $\Delta^{*}$. Hence, in this case $\Delta^{*}$ is indeed the optimal choice for the investor.

This is no longer the case in the right plot. Here the marginal benefit curve intersects the marginal cost curve three times (at $\Delta_{1}^{*}, \Delta^{*}$, and $\Delta_{2}^{*}$ ). Since the marginal benefit is below the marginal cost for values of $\Delta$ that are smaller and "close" to $\Delta^{*}$, while the marginal benefit is larger than the marginal cost for values of $\Delta$ that are larger and "close" to $\Delta^{*}, \Delta^{*}$ is a local minimum, and hence a suboptimal choice. By contrast the points $\Delta_{1}^{*}$ and $\Delta_{2}^{*}$ are local maxima. (In this particular example the point $\Delta_{1}^{*}$ is the global maximum, since the area $A$ is larger than the area $B$.) The fact that $\Delta^{*}$ is not a maximum implies that there does not exist a symmetric equilibrium, since in a symmetric equilibrium it would have to be the case that $w^{f}=0$.

The non-existence of a symmetric market equilibrium implies that one should look for equilibria where investors in the same location make different choices, even though they have 


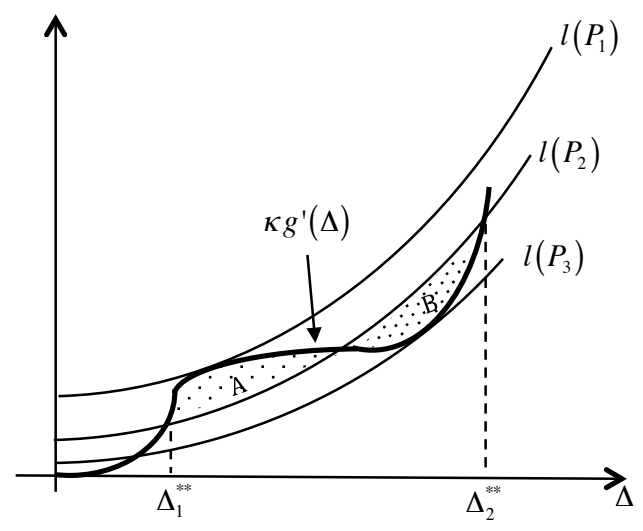

Figure 7: Illustration of an asymmetric equilibrium involving mixed strategies. The price adjusts so that area $A$ is equal to area $B$. Accordingly, an investor is indifferent between $\Delta_{1}^{* *}$ and $\Delta_{2}^{* *}$. Here, $P_{3}<P_{2}<P_{1}<1$.

the same preferences, endowments, and information. Figure 7 presents a simple graphical illustration of such an equilibrium in the context of the example depicted on the right plot of Figure 6.

Specifically we illustrate the construction of an equilibrium that features the same price $P_{i}=P$ for all markets, but where a fraction $\pi$ of investors in every location $i$ choose $\left(w_{1}^{f}, \Delta_{1}\right)$, while the remaining fraction $(1-\pi)$ of agents choose $\left(w_{2}^{f}, \Delta_{2}\right)$. We refer to such an equilibrium as an asymmetric, location-invariant equilibrium. We introduce a function $l$ that captures the marginal benefit of participation as a function of $\Delta$ and $P$ :

$$
l(\Delta, P) \equiv-\frac{\gamma}{2}\left(1-w^{f}(\Delta, P)\right)^{2} \omega^{\prime}(\Delta)=-\frac{\gamma}{2}\left(\frac{1-P}{\gamma}\right)^{2} \frac{\omega^{\prime}(\Delta)}{\omega^{2}(\Delta)}
$$

where the last equation follows from (18).

Figure 7 depicts the function $l(\Delta, P)$ for three values $P_{1}<P_{2}<P_{3}<1$. A first observation is that as $P$ declines from $P_{3}$ to $P_{1}$, the curve $l(\Delta, P)$ shifts up. Moreover there exists a level $P_{2}$ and associated values $\Delta_{1}^{* *}$ and $\Delta_{2}^{* *}$ for which the area "A" equals the area "B", so that investors are indifferent between choosing $\Delta_{1}^{* *}$ and $\Delta_{2}^{* *}$. Fixing these values of $P, \Delta_{1}^{* *}$, and $\Delta_{2}^{* *}$, we can determine the values of $w_{1}^{f}$ and $w_{2}^{f}$ from (18). As part of the proof of Proposition 2 (in the appendix), we show that these values of $w_{1}^{f}$ and $w_{2}^{f}$ satisfy $1-w_{1}^{f}<1<1-w_{2}^{f}$. In order to clear the bond market, $\pi$ has to be so that $\pi w_{1}^{f}+(1-\pi) w_{2}^{f}=0$, i.e., $\pi=\frac{w_{2}^{f}}{w_{2}^{f}-w_{1}^{f}} \in(0,1) .{ }^{14}$ We also show in the appendix that for this value of $\pi$ all markets for risky assets clear as well.

The next proposition generalizes the insights of the above illustrative example. It states

\footnotetext{
${ }^{14} \pi \in(0,1)$ since $w_{2}^{f}<0$ and $w_{1}^{f}>0$
} 
the existence of an asymmetric, location-invariant equilibrium when a symmetric equilibrium fails to exist.

Proposition 2 When the cost function $g$ is such that a symmetric equilibrium fails to exist, there exists an asymmetric, location-invariant equilibrium. Specifically, there exist (at least) two tuples $\left\{\Delta_{k}, w_{k}^{f}\right\}, k \geq 2$, and $\pi_{k}>0$ with $\sum_{k} \pi_{k}=1$ and $\sum_{k} \pi_{k}\left(1-w_{k}^{f}\right)=1$, such that in every location $i$ a fraction $\pi_{k}$ of agents choose the interval, leverage, and portfolio combination $\left\{\Delta_{k}, w_{k}^{f}, d L^{(k)}\right\}$, where $d L^{(k)}$ is the measure given in (12) for $\Delta=\Delta_{k}$. Finally, a symmetric and an asymmetric location-invariant equilibrium cannot co-exist. ${ }^{15}$

The model can therefore generate situations in which a symmetric equilibrium can fail to exist. Instead, the market equilibrium features a diverse set of financial strategies, with some investors pursuing high-cost, high-Sharpe-ratio, high-leverage strategies and some investors pursuing low-cost, low-Sharpe-ratio, no-leverage strategies. The first type of strategies is akin to the sort of strategies offered by hedge funds, while the second type of strategies is akin to those offered by mutual funds.

A novel feature of our model is that it helps isolate a source of non-concavity of the participation decision that is distinct from non-convex participation cost structures. Specifically, the continuity of the dividend structure in our framework along with the convexity of $g(\Delta)$ allow us to model the participation choice (i.e., the choice of $\Delta$ ) as a concave decision problem for fixed leverage. Accordingly, we can illustrate the role of the complementarity between leverage and participation in rendering the participation problem non-concave. The distinct role of this complementarity can be easily overlooked in models with discrete markets where the participation choice itself (i.e. even with fixed leverage) is discrete and hence necessarily non-convex.

Clearly, if we were to introduce elements of non-convexity into the cost structure (for instance, because investors may choose non-contiguous participation locations, as in the next section or in Appendix A.2), there is an additional and conceptually distinct reason why the investor's objective is non-concave, leading to the adoption of diverse financial strategies. ${ }^{16}$

In summary, this section has established that the non-concavities in investors' objective functions introduce diversity in the optimal participation-leverage combinations chosen by different investors. Importantly, the equilibrium price plays a key role in determining the participation-leverage combinations that keep investors indifferent between different strategies. In the next section we identify a tension that arises when we introduce collateral con-

\footnotetext{
${ }^{15}$ As part of the proof of the proposition we also show that asymmetric, location-invariant equilibria are essentially unique: All asymmetric equilibria have the same asset prices.

${ }^{16}$ A classic example, which also features different choices by ex-ante identical investors, due to fixed (hence non-convex) information costs, is Grossman and Stiglitz (1980). A difference between Grossman and Stiglitz (1980) and our setup, which becomes pertinent in the next section, is that the price adjusts to keep investors indifferent between different combinations of leverage and participation, while in Grossman and Stiglitz (1980) investors decide on paying information acquisition costs before observing the price.
} 
straints, so that the price simultaneously affects investors' indifference relations and their feasible leverage-participation combinations.

\section{Collateral Constraints and Price Crashes}

The previous section shows that equilibrium outcomes may feature heterogeneous investment strategies in every location despite the fact that investors are identical. An important aspect of these heterogeneous strategies is that they require different degrees of leverage. Even though in the model leverage can be chosen freely, in reality leverage is limited by collateral constraints. In this section we discuss the implications of such constraints. We present a simple example to show that leverage constraints open the possibility for multiple locationinvariant equilibria. Accordingly, prices, capital flows, and leverage exhibit discontinuous dependence on incremental changes to participation costs.

To model collateral constraints, we let $\chi \in[0,1)$ denote a "haircut" parameter and require

$$
\left[(1-\chi) \int_{0}^{1} P_{j} d X_{j}^{(i)}-F_{i}\right]+X_{B}^{(i)} \geq 0 .
$$

Equation (23) stipulates that the borrowing capacity of an investor (the first term - inside the square brackets - of (23)) do not exceed the amount the investor actually borrows (the second term of (23)). The investor's borrowing capacity is computed as the market value of the collateral net of the haircut and of the obligation the investor has already assumed by committing to pay the participation costs in period 1.

The collateral constraint (23) is pervasive in reality, extensively used in the literature, and analytically convenient for our purposes. Therefore, we focus on the implications of this constraint in the body of the paper. In Appendix D we analyze an alternative, endogenous version of the constraint (23) motivated by the desire to prevent default in all states of nature. We show that the key insights of the present section remain the same.

To further streamline the exposition, we choose a particularly simple specification for the participation cost structure that includes a discrete element of participation choice. This choice introduces an additional and direct reason for the existence of asymmetric equilibria featuring heterogenous leverage. More importantly, it allows us to provide an analytically solvable example of participation- and leverage- choice that helps illustrate the role of leverage constraints when leverage and participation choices interact.

Specifically, we postulate the cost function depicted in the middle graph of Figure 4. In that case, the optimal participation choice amounts to choosing the location of the points $a_{i}$ and the number of the discrete points $N .{ }^{17}$ To keep computations as simple as possible, we assume furthermore that $b_{N}=\infty$ for $N>2$, so that only choices involving $N \leq 2$ are

\footnotetext{
${ }^{17}$ Recall that in this case there is a minimum "fixed" cost that one needs to pay for every new location that she chooses given by $\kappa b_{N} \lim _{x \rightarrow 0} f(x)>0$.
} 
feasible. ${ }^{18}$ Accordingly, an investor's problem comes down to choosing $N=1$ (and paying no participation costs) or $N=2$ and the distance $d$ from her home location, resulting in participation costs equal to $\kappa f(d)$. (See Appendix A.2 for an extension to multiple locations.) In an effort to further streamline the analysis, we assume that $f^{\prime}(d)=0$. In that case the investor faces only a fixed cost of $\kappa f_{0}$ when choosing $N=2$, but can choose $d$ freely (i.e., without incurring additional cost).

We start by analyzing equilibrium prices in this economy when we don't impose the constraint (23) on investors' decisions.

Proposition 3 Assume that the borrowing constraint is not imposed on investors' decisions, and also assume that $\kappa \in\left(\kappa_{1}, \kappa_{2}\right)$, where $\kappa_{1} \equiv \frac{1}{128} \frac{\gamma \sigma^{2}}{f_{0}}, \kappa_{2} \equiv \frac{1}{8} \frac{\gamma \sigma^{2}}{f_{0}}$. Then there exists a unique asymmetric equilibrium in which a fraction $\pi>0$ of the investors choose $N=1$, while the rest choose $N=2$ and $d=\frac{1}{2}$. The equilibrium price in all locations is

$$
P(\kappa)=1-\sigma \sqrt{\frac{\gamma \kappa f_{0}}{18}}
$$

and the choices $w_{i}^{f}$ satisfy $w_{1}^{f}>0$ and $w_{2}^{f}<0$.

For values of $\kappa$ smaller than $\kappa_{1}$ or larger than $\kappa_{2}$ the equilibrium is symmetric (and unique in the class of location-invariant equilibria) with everyone choosing $N=2$ and $d=\frac{1}{2}$ or everyone choosing $N=1$, respectively. Hence, without imposing the constraint (23), the left plot of Figure 8 gives a visual depiction of $P(\kappa)$, which is a continuous function of $\kappa$.

When imposing the constraint (23), however, a different situation arises: For any given value of $\kappa$ there may be multiple equilibria. A direct implication of equilibrium multiplicity is that it may become impossible to find a continuous mapping $\kappa \rightarrow P(\kappa)$. Such a situation is depicted in the right plot of Figure 8, which graphs the equilibrium price in the absence of constraints, the locus of points where the constraint is binding, and the maximum price associated with an equilibrium when the constraint is binding.

To understand the source of multiple equilibria and why they imply the non-existence a continuous mapping $\kappa \rightarrow P(\kappa)$, it is useful to refer to Figure 9. For each subplot we fix a value of $\kappa$. Given that value of $\kappa$, the line denoted "Indifference" in each subplot depicts combinations of $P$ and possible choices of $\left(1-w_{2}^{f}\right)$ such that the indifference relation $V_{1}=V_{2}$ holds. ${ }^{19}$ Similarly, the line denoted "Constraint" depicts combinations of $P$ and $1-w_{2}^{f}$ such

\footnotetext{
${ }^{18}$ The condition $b_{N}=\infty$ can be substantially weakened. Indeed all that is needed for $N=1$ or $N=2$ to be the only feasible choices is that $\kappa b_{N} \lim _{x \rightarrow 0} f(x)>1$ for $N>2$. To see this, use the normalization $P_{B}=1$ along with the budget constraint to obtain $P_{i}=W_{0, i}=\int_{0}^{1} P_{j} d X_{j}^{(i)}+X_{B}^{(i)}=(1-\chi) \int_{0}^{1} P_{j} d X_{j}^{(i)}-$ $F_{i}+X_{B}^{(i)}+F_{i}+\chi \int_{0}^{1} P_{j} d X_{j}^{(i)} \geq F_{i}+\chi \int_{0}^{1} P_{j} d X_{j}^{(i)}$, where the inequality follows from (23). Noting that $P_{j} \leq 1$ and $f$ is non-decreasing, the only feasible choices are $N=1$ or $N=2$ if $\kappa b_{N} \lim _{x \rightarrow 0} f(x)>1$ for $N>2$.

${ }^{19}$ See equation (47) in the appendix for a formal statement of the indifference relation.
} 

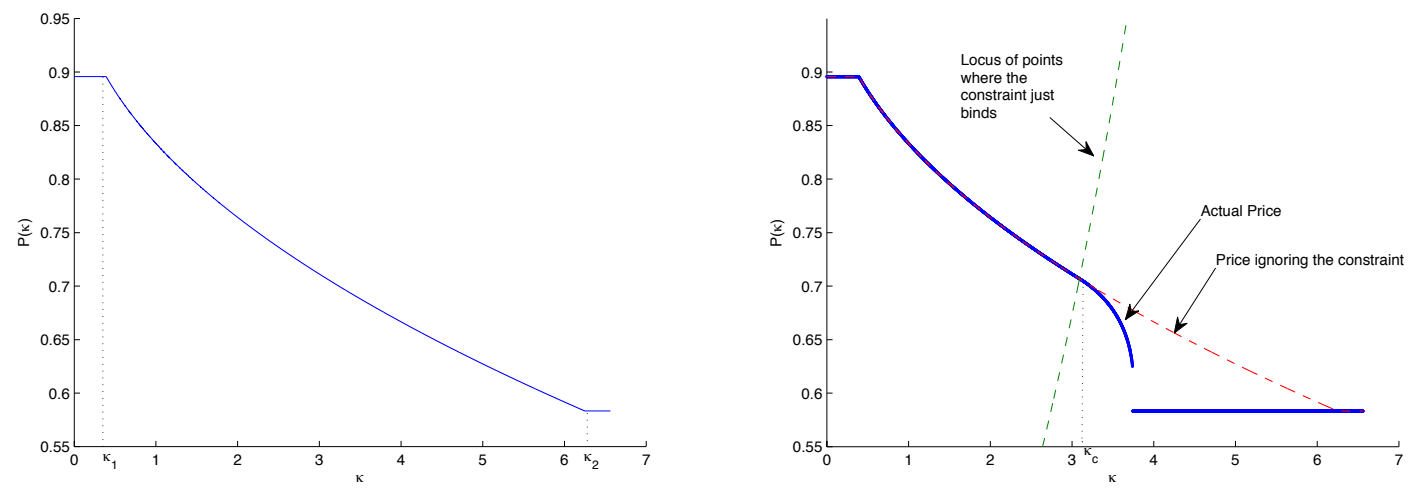

Figure 8: The left plot depicts the price $P(\kappa)$ assuming the leverage constraint is not binding. The downward-sloping dotted line on the right plot plots the same quantity, along with the actual price (solid line) and the locus of points such that the leverage constraint is just binding (upward sloping line). Hence for points above and to the left of the upward sloping line the constraint is not binding, whereas it is binding for points below and to the right. Parameters used in this example: $\gamma=5, f_{0}=0.1, \chi=0.2, \sigma=1$.

that the constraint (23) binds with equality, i.e., the set of points such that ${ }^{20}$

$$
\kappa f_{0}=P\left(1-\chi\left(1-w_{2}^{f}\right)\right)
$$

Accordingly, all the points that lie above that line are admissible combinations of $P$ and $1-w_{2}^{f}$. The line denoted "FOC" (in the first plot) depicts the combination of points that satisfy the (unconstrained) first-order condition for leverage

$$
1-P=\gamma\left(1-w_{2}^{f}\right) \omega^{(N=2)}
$$

where $\omega^{(N=2)}=\frac{\sigma^{2}}{48}$ is the minimal variance attainable for an investor choosing to participate in $N=2$ locations. Finally, the line denoted "Lowest Price" is a reference line denoting the price that would obtain in the symmetric equilibrium where all investors participate exclusively in their own location.

The left plot depicts the case $\kappa=\kappa_{c}$. In that case the three lines "FOC", "Indifference", and "Constraint" intersect at the same point (C). This reflects that when $\kappa=\kappa_{c}$, the equilibrium combination of price and optimal leverage for an investor choosing $N=2$ are such that the leverage constraint is just binding. Moreover, point $\mathrm{C}$ is the unique equilibrium.

For (moderately) higher values of $\kappa$, illustrated in the middle panel, the constraint binds actively, so that the first order condition for the optimal choice of leverage no longer holds

\footnotetext{
${ }^{20}$ Combining the investor's budget constraint with (23) and repeating the same steps as in footnote 18 gives $W_{0, i} \geq F_{i}+\chi \int_{0}^{1} P_{j} d X_{j}^{(i)}$. Evaluating this inequality with $P_{i}=P_{j}=P=W_{0, i}, P_{B}=1, F_{i}=\kappa f_{0}$, and $\int_{0}^{1} d X_{j}=1-w^{f}$ gives (25).
} 

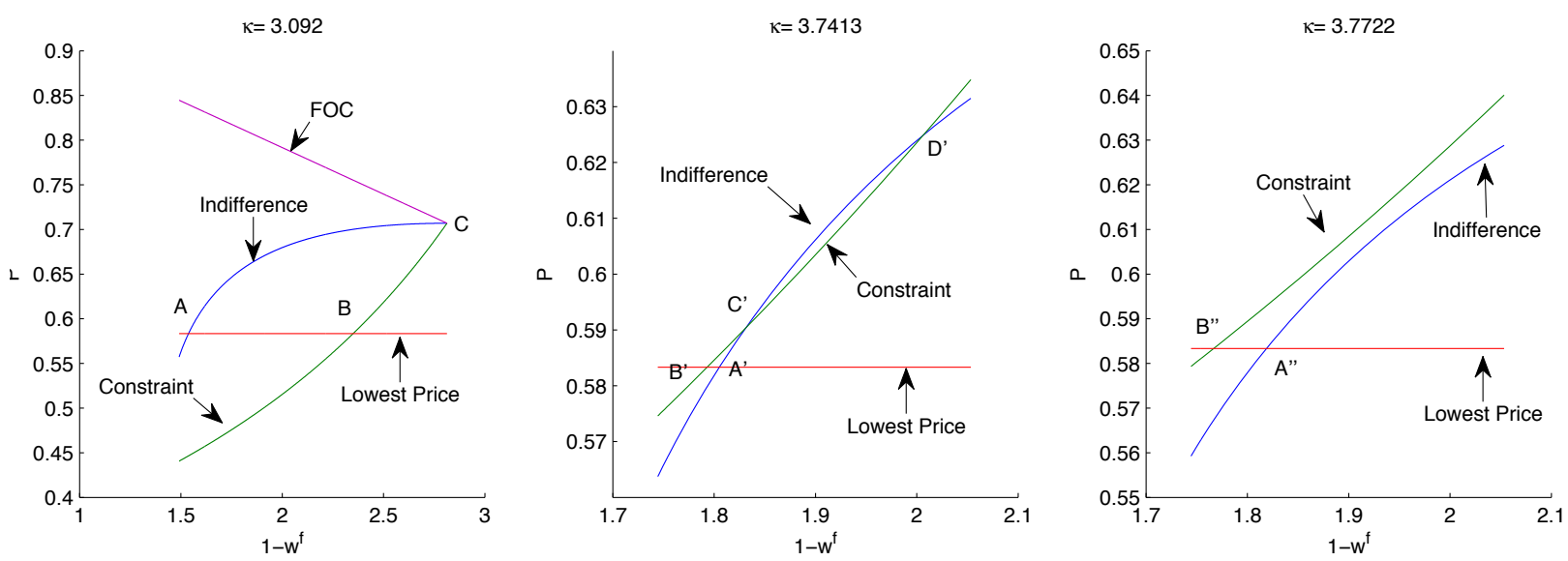

Figure 9: For three different values of the cost parameter $\kappa$, the three plots depict combinations of price $(P)$ and leverage $\left(1-w_{2}^{f}\right)$ so that (i) investors are indifferent between adopting no- and high-leverage strategies (line labeled "Indiference"); (ii) investors' choices of leverage $\left(1-w_{2}^{f}\right)$ are (unconstrained) optimal given $P$ (line labeled "FOC"); and (iii) the leverage constraint just binds (line labeled "Constraint"). For reference, we also plot the equilibrium price associated with all investors participating exclusively in their own location (line labeled "Lowest price"). Parameters are identical to Figure 8.

with equality. Instead, candidate asymmetric equilibria are given by the intersection of "Indifference" and "Constraint". Clearly, there are two such equlibria (points C' and D'). The symmetric equilibrium whereby everyone chooses $N=1$ is yet another equilibrium. (To see this, note that point $\mathrm{A}^{\prime}$ is to the right of point $\mathrm{B}^{\prime}$. This implies that if all investors choose $N=1$ and zero leverage, then an individual investor would not benefit by deviating to $N=2$; if she did deviate, her leverage would be given by the point $\mathrm{B}^{\prime}$, which is above and to the left of the line labeled "Indifference" - hence inferior to choosing $N=1$ ).

The right-most plot shows a case in which $\kappa$ is large enough that there is no point of intersection between the lines "Indifference" and "Constraint". At that point there can be no asymmetric equilibriua. The equilibrium becomes symmetric, i.e., the price drops to the level that obtains when everyone chooses $N=1$ and $w^{f}=0$.

The multiplicity of equilibria implies that the mapping $\kappa \rightarrow P(\kappa)$ is discontinuous at some value $\kappa>\kappa_{c}$, no matter how one chooses the equilibrium. ${ }^{21}$ The fact that multiplicity of equilibria implies a discontinuous mapping $\kappa \rightarrow P(\kappa)$ is reminiscent of Gennotte and Leland (1990), albeit in a different context.

To understand intuitively why there are multiple equilibria when the leverage constraint

\footnotetext{
${ }^{21}$ For instance if one chooses the equilibrium associated with the maximal price, then the discontinuity will occur for some value of $\kappa$ between those depicted in the middle and the right-most panel of Figure 9 . Similarly, if one chooses the equilibrium with the minimal price, then the jump will occur at some value of $\kappa$ between those depicted in the left-most and middle graph. More generally, it is impossible to find a choice of equilibrium such that $P(\kappa)$ is continuous for the values of $\kappa$ between the left-most and the right-most plots.
} 
is binding, it is useful to start with the case when the constraint is not binding. In that case the incentives of investors to pursue high-participation strategies are substitutes: The less investors choose to participate in such strategies, the lower the prices of risky assets, and thus the higher an individual investor's incentive to pursue such strategies. As is commonly the case when economic decisions are substitutes, the equilibrium is unique and features a price that keeps investors indifferent between high- and low-participation strategies.

A different situation arises when the leverage constraint binds. Reductions in prices increase the Sharpe ratio of risky strategies, but also constrain the ability to leverage. Since leverage and participation choices are complements, a decline in price may end up lowering rather than increasing the incentive to participate, rendering the choices to pursue high-participation strategies complements rather than substitutes. As a result there can be multiple equilbria, which in turn imply discontinuous dependence of $P(\kappa)$ on $\kappa$.

We note that even though leverage constraints are featured in several papers in the literature, our analysis helps highlight how the complementarity between leverage and participation may result in making entry decisions complements rather than substitutes. Appendix D shows that this discontinuity survives in an extension of the model with leverage constrained endogenously (in an effort to prevent default).

\section{Reduced Participation in a Subset of Locations, En- tanglement, and Contagion}

In the previous sections we assumed the same cost structure for all investors, regardless of location. This allowed us to focus on (potentially asymmetric but) location-invariant equilibria, which entail the same price in all locations. In this section we relax this assumption in order to study the effect of an adverse shock to financial participation in a subset of locations. The challenge in analyzing the model with location-specific costs is that an entire price function on $[0,1)$ has to be computed, rather than a single value. To present the key insights of the model with location-specific costs, we restrict ourselves to a specific setup that is sufficiently simple to analyze, yet rich enough to illustrate how a reduction in market participation in a subset of risky markets can propagate across all markets.

Specifically, we consider the following stylized setup: for some positive $k<1$ market access in locations $\left[-\frac{k}{2} ; \frac{k}{2}\right]$ "breaks down". Specifically, we assume that in these "affected" locations the cost parameter $\kappa$ becomes infinite, so that investors choose to participate only in the bond market and the market for the local risky claim, but not in other markets. In the rest of the locations investors choose to participate in a single interval of length $\bar{\Delta}$, centered at their "home location" - this would be the outcome of a cost structure in these locations involving, e.g., $\kappa<\infty, b_{N}=\infty$ for $N>1, g^{\prime}(\bar{\Delta})=0$ for $\Delta<\bar{\Delta}$, and $g^{\prime}(\Delta)$ sufficiently large for $\Delta>\bar{\Delta}$.

We solve this heterogeneous-participation-costs version of the model in the appendix. 


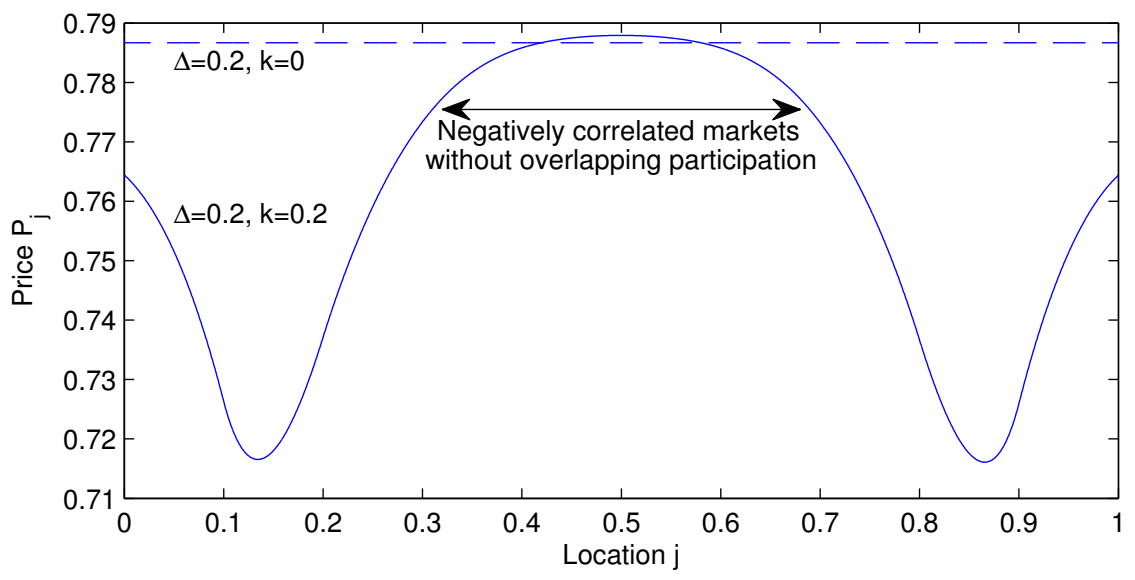

Figure 10: Contagion effects. The price function $P_{j}$ depicts the price in different locations when $k=0$ and $k=0.2$. We set the length of the participation arc of "unaffected" investors to $\Delta=$ $\bar{\Delta}=0.2$. The locations between the arrows feature both negative correlation of dividends and no overlap between the participants in these markets and the set $A=\left[-\frac{k}{2}, \frac{k}{2}\right]$.

First, we calculate analytically the optimal demand of every investor given a price function (Lemma 3), and then aggregate the demands to solve for prices. We are unable to find a closed-form solution for the prices, but we can characterize the solution in terms of a linear integro-differential equation with delay, which can be solved numerically as easily as a matrix inversion problem.

To obtain a visual impression of the solution, Figure 10 depicts the price $P_{j}$ for $j \in[0,1]$ and compares it with the (symmetric) equilibrium price $P^{*}(\bar{\Delta})$ that would prevail if all agents in all locations chose a participation interval with length equal to $\Delta=\bar{\Delta}$. There are several noteworthy facts about Figure 10. As one might expect, prices in the set $A \equiv\left[-\frac{k}{2}, \frac{k}{2}\right]$ are lower than $P^{*}(\bar{\Delta})$. The more important fact is that prices in all other markets are affected, as well. This holds true even in markets in the interval $B \equiv\left[\frac{k}{2}+\bar{\Delta}, 1-\frac{k}{2}-\bar{\Delta}\right]$, i.e., in locations that are at a larger distance than $\bar{\Delta}$ from the set $A$, so that agents in the set $A$ would not be directly trading risky assets with agents in the set $B$, even if they were allowed to choose the same participation interval $\bar{\Delta}$ as everyone else. A third observation is that the prices in almost all markets are lower than $P^{*}(\bar{\Delta})$. Indeed, this reduction in price can happen even in markets with dividends that have a zero or negative correlation with the dividends of any risky security in $A$, as the figure illustrates.

The intuition behind Figure 10 is the following. By assumption, investors in the set $A$ concentrate all their demand for risky assets in their home location. Hence, compared to the case in which everyone participates in an interval of length $\bar{\Delta}$ centered at their location, there is now lower demand for risky securities in locations neighboring the set $A$. The lower demand for risky securities in these locations leads to lower prices, which attracts demand from locations adjacent to the neighborhood of $A$. By tilting their portfolio towards 
these locations, investors remove demand for risky securities in locations even farther from $A$. Accordingly, prices in these farther locations need to drop in order to attract investors from locations adjacent to the neighbourhood of the neighbourhood of $A$, who also tilt their portfolios and so on.

This chain reaction implies that all locations are affected in equilibrium. In fact, even some prices in locations close to point $\frac{1}{2}$, the farthest from $A$, drop, as the following result states. $^{22}$

Proposition 4 Assume $\bar{\Delta}+k<1$ and that $\frac{1}{2}=\arg \max _{j \in\left[\frac{1}{2}-\bar{\Delta}, \frac{1}{2}+\bar{\Delta}\right]} P_{j}$. Then there exists a positive-measure subset $D \subset\left[\frac{1}{2}-\bar{\Delta} ; \frac{1}{2}+\bar{\Delta}\right]$ such that $P(x)<P^{*}(\bar{\Delta})$ for all $x \in D$.

Proposition 4 helps formalize the notion that the drop in prices caused by restricting market access for investors in the set $A$ can permeate even a neighborhood of radius $\bar{\Delta}$ around the maximal-price location.

The extent of the change in risk premia in the various locations depends on the distance from the $\operatorname{arc} A$, the length of the $\operatorname{arc} A$, and most interestingly, on the length of the participation arc $\bar{\Delta}$. Figure 11 illustrates these statements. If $\bar{\Delta}$ is small, so that investors' portfolios are heavily exposed to risks in their vicinity, then even a small portfolio tilt towards a nearby location requires a relatively high risk compensation. By contrast, if investors' portfolios are invested across a broad range of locations, then they are more willing to absorb risks in their vicinity.

In summary, a surprising result of our analysis is that contagion occurs due to limited, rather than excessive, integration of risky markets. Indeed, contagion may become stronger the weaker the integration of risky markets. Finally, in model simulations not reported here due to space limitations, we have also considered the effect of local participation shocks when investors participate in multiple disjoint intervals, obtaining similar conclusions. ${ }^{23}$

We conclude this section by noting that the location-dependent version of the model has implications beyond the contagion ones we highlighted here. For instance, the complementarity between leverage and participation means that large arcs of participation and high degrees of leverage tend to be observed in the same locations. ${ }^{24}$ Thus, countries with superior participation technologies (e.g., due to a better financial industry) have higher leverage. This model prediction appears consistent with the evidence that a country such as the US

\footnotetext{
${ }^{22}$ It is easy to show that, in the general case when a subset of investors are limited to a smaller investment set than the original arc of length $\bar{\Delta}$, and therefore in the setting of this section, the average risky-asset price in the economy declines.

${ }^{23}$ In such situations, the direct impact of a local shock to participation is felt not only in the neighboring locations of the affected locations, but also in the other subintervals where the affected investors used to participate. This sets off multiple "waves" of contagion across the circle (i.e., Figure 11 features more than two local minima).

${ }^{24}$ For instance, in the example of this section the locations in the set $A=\left[-\frac{k}{2}, \frac{k}{2}\right]$, where participation in risky markets is impaired, exhibit positive holdings of bonds.
} 

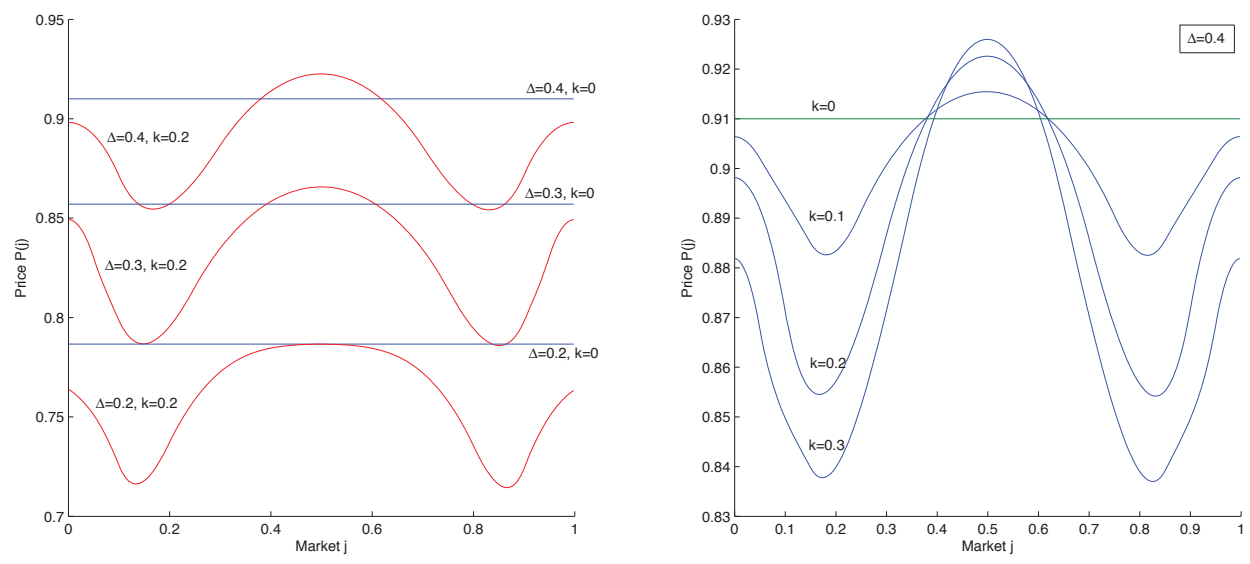

Figure 11: Contagion effects for various choices of $\bar{\Delta}$ and $k$. In the figures $j$ denotes a specific market, $k$ controls the length of the region with restricted access to markets, while $\Delta$ refers to $\bar{\Delta}$, that is the length of the participation arc for investors who can participate in "distant" markets.

has a riskier portfolio of overseas assets than a country such as China, while the US is a borrower in bond markets, as opposed to China, who is a lender.

\section{Conclusion}

We introduce a novel, location-invariant, and tractable structure of shocks on a circle that allows us to model the tradeoffs between integration and risk diversification on the one hand and participation costs on the other. The focus of the analysis is on the private incentives to incur costs to participate in various asset markets, and on the interdependence ("entanglement") of markets in the presence of limited integration.

Our main findings can be grouped in three broad categories. First, participation decisions and leverage decisions are complements. Investors who choose to participate in a large number of markets also choose high degrees of leverage. The interaction between leverage decisions and participation routinely makes an investor's optimization problem non-concave. Such non-concavities may imply a heterogeneous market structure. Despite the fact that all investors are identical, and even when participation costs are convex, some investors may opt for non-levered, low-participation investment strategies with others opting for levered, high-participation strategies.

Second, the non-concavity of an investor's participation problem can lead to an abrupt change in the nature of equilibrium. Even small variations in participation costs can lead to rapid drops in leverage, prices and market integration when the ability to leverage is limited by the availability of collateral.

Third, asset markets exhibit increased interdependence despite - in fact, because of limited market integration. Limitations to market access in one subset of locations propa- 
gates across markets. Surprisingly, the impact of these local shocks on other markets may be strengthened when markets are less integrated. In an intertemporal version of the model, the joint determination of market integration and equilibrium prices implies price and return correlations that exceed those of the underlying dividends. 


\section{References}

Acemoglu, D., A. Ozdaglar, and A. Tahbaz-Salehi (2013). Systemic risk and stability in financial networks. Working Paper, MIT.

Ahrend, R. and C. Schwellnus (2012). Do investors disproportionately shed assets of distant countries under increased uncertainty? evidence from the global financial crisis. Working Paper, OECD Economics Department.

Allen, F., A. Babus, and E. Carletti (2012). Asset commonality, debt maturity and systemic risk. Journal of Financial Economics 104, 519-534.

Allen, F. and D. Gale (2000). Financial contagion. Journal of Political Economy 108, 1-33.

Aumann, R. J. (1966). Existence of competitive equilibria in markets with continuum of traders. Econometrica 34, 1-17.

Aviat, A. and N. Coeurdacier (2007). The geography of trade in goods and asset holdings. Journal of International Economics 71, 22-51.

Barlevy, G. and P. Veronesi (2003). Rational panics and stock market crashes. Journal of Economic Theory 110, 234-263.

Bekaert, G. and C. Harvey (1995). Time-varying world market integration. Journal of Finance 50, 403-444.

Brunnermeier, M. K. and L. H. Pedersen (2009). Market liquidity and funding liquidity. Review of Financial Studies 22, 2201-2238.

Buch, C. M. (2005). Distance and international banking. Review of International Economics 13(4), 787-804.

Caballero, R. J. and A. Simsek (2012). Fire sales in a model of complexity. forthcoming, Journal of Finance.

Chan, K., V. Covrig, and L. Ng (2005). What determines the domestic bias and foreign bias? evidence from mutual fund equity allocations worldwide. Journal of Finance 60 (3), 1495-1534.

Cochrane, J. H., F. A. Longstaff, and P. Santa-Clara (2008). Two trees. Review of Financial Studies 21, 347-385.

Coval, J. D. and T. J. Moskowitz (1999). Home bias at home: Local equity preference in domestic portfolios. Journal of Finance 54, 1695-1704.

Coval, J. D. and T. J. Moskowitz (2001). The geography of investment: Informed trading and asset prices. Journal of Political Economy 109, 811-841. 
Freixas, X., B. Parigi, and J.-C. Rochet (2000). Systemic risk, interbank relations and liquidity provision by the central bank. Journal of Money, Credit and Banking 32, 611638.

Gârleanu, N., S. Panageas, and J. Yu (2013). Impediments to financial trade: Theory and measurement. Working Paper.

Gennotte, G. and H. Leland (1990). Market liquidity, hedging and crashes. American Economic Review 80, 999-1021.

Giannetti, M. and L. Laeven (2012). The flight home effect: Evidence from the syndicated loan market during financial crises. Journal of Financial Economics 104, 23-43.

Grinblatt, M. and M. Keloharju (2001). How distance, language, and culture influence stockholdings and trades. Journal of Finance 56(3), 1053-1073.

Grossman, S. J. and J. E. Stiglitz (1980, June). On the impossibility of informationally efficient markets. American Economic Review 70(3), 393-408.

Head, K. and J. Ries (2008). FDI as an outcome of the market for corporate control: Theory and evidence. Journal of International Economics 74, 2-20.

Hoggarth, G., L. Mahadeva, and J. Martin (2010). Understanding international bank capital flows during the recent financial crisis. Financial Stability Paper No. 8, Bank of England.

Huberman, G. (2001). Familiarity breeds investment. Review of Financial Studies 14(3), $659-680$.

Kiyotaki, N. and J. Moore (1997). Credit cycles. Journal of Political Economy 105, 211-248.

Krishnamurthy, A. (2010). Amplification mechanisms in liquidity crises. American Economic Journal: Macroeconomics 2(3), 1-30.

Kyle, A. S. and W. Xiong (2001). Contagion as a wealth effect. Journal of Finance 56(4), $1401-1440$.

Merton, R. C. (1987). A simple model of capital market equilibrium with incompletel information. Journal of Finance 42, 483-510.

Pavlova, A. and R. Rigobon (2008). The role of portfolio constraints in the international propagation of shocks. Review of Economic Studies 75, 1215-1256.

Portes, R. and H. Rey (2005). The determinants of cross-border equity flows. Journal of International Economics 65, 269-296.

Salop, S. C. (1979). Monopolistic competition with outside goods. Bell Journal of Economics 10, 141-156. 
Talamo, G. (2007). Institutions, FDI, and the gravity model. Working Paper, Universita de Palermo.

Vila, J.-L. (1989). Simple games of market manipulation. Economics Letters 29(1), 21-26.

Yuan, K. (2005). Asymmetric price movements and borrowing constraints: A rational expectations equilibrium model of crises, contagion, and confusion. Journal of Finance 60, 379-412.

Zawadowski, A. (2013). Entangled financial systems. Review of Financial Studies 26, 12911323. 


\section{Appendix}

\section{A Extensions}

\section{A.1 An infinite horizon version of the baseline model}

In this section we develop an intertemporal version of the model. The intertemporal version allows us to extend the intuitions of our comparative statics exercises to a framework where the shocks to the participation technology are recurrent.

Specifically, we keep the key assumptions of the baseline model (Section 4.2), and in particular the assumption that investors only participate in an arc surrounding their home location. However, we assume that investors maximize expected discounted utility from consumption

$$
-\sum_{t=0}^{\infty} \beta^{t} E_{0}\left[e^{-\gamma c_{t, i}}\right],
$$

where $t$ denotes (discrete) calendar time. We assume that dividends at location $i \in[0,1)$ are given by

$$
D_{t, i}=(1-\rho) \sum_{k=-\infty}^{t} \rho^{t-k} \varepsilon_{k, i}
$$

where $\rho<1$,

$$
\varepsilon_{t, i} \equiv 1+\sigma\left(B_{i}^{(t)}-\int_{0}^{1} B_{j}^{(t)} d j\right)
$$

and $B_{i}^{(t)}$ denotes a family of Brownian Bridges on [0,1] drawn independently across times $t \in$ $\{-\infty, \ldots, 1,2, \ldots, \infty\}$.

We make a few observations about this dividend structure. First, we note that equation (27) coincides with equation (3). Accordingly, $\int_{0}^{1} \varepsilon_{t, i} d i=1$, and therefore, equation (26) implies that $\int_{0}^{1} D_{t, i}=1$ for all $t$, so that the aggregate dividend is always equal to one. Second, dividends at individual locations follow $\mathrm{AR}(1)$ processes, since equation (26) implies

$$
D_{t, i}=(1-\rho) \varepsilon_{t, i}+\rho D_{t-1, i} .
$$

Moreover, since (27) coincides with (3), the increments of two dividend processes at two locations $i$ and $j$ have the covariance structure of equation (4).

In terms of participation decisions, we keep the same cost assumption as in the baseline model and further assume that investors participate in a single interval of length $\Delta_{t}^{(i)}$ centered at their "home" location. Participation costs are paid period by period in advance of trading. Specifically, an investor's intertemporal budget constraint is given by

$$
c_{t, i}+F_{t}\left(\Delta_{t}^{(i)}\right)+\int_{0}^{1} P_{t, j} d X_{t, j}^{(i)}+P_{B, t} X_{B, t}^{(i)}=\int_{0}^{1}\left(P_{t, j}+D_{t, j}\right) d X_{t-1, j}^{(i)}+X_{B, t-1}^{(i)} .
$$

We note that in equation (29) we allow the entire cost function $F_{t}(\Delta)=\kappa_{t} g_{t}(\Delta)$ to be differ- 
ent across different periods in order to capture the effect of repeated shocks to the participation technology.

This intertemporal version of the model presents a challenge that is absent in a static framework: If the interest rate varies over time, then the value function of an agent is not exponential in wealth. In fact, a closed-form expression for the value function most likely does not exist. Furthermore, once the value function is no longer exponential, portfolios are no longer independent of wealth, and hence the entire wealth distribution matters - an infinite-dimensional state variable.

In order to maintain the simple structure of the solution, therefore, we make necessary assumptions to achieve a constant interest rate in the presence of random participation costs, while safeguarding market clearing for bond markets, risky-asset markets, and consumption markets. ${ }^{25}$ The following proposition states that it is possible to achieve this outcome by judiciously specifying the distribution of the participation costs. The main thrust of the proposition, however, concerns the expression for the risky-asset prices.

Proposition 5 There exist an interval $\left[\Delta_{l}, \Delta_{u}\right]$, a (non-trivial) distribution function $\Psi(\cdot)$ on $\left[\Delta_{l}, \Delta_{u}\right]$, and a cost function $F\left(\cdot ; \Delta_{t}\right):\left[\Delta_{l}, \Delta_{u}\right] \rightarrow \mathbb{R}^{+}$such that, if $\Delta_{t}$ is drawn in an i.i.d. fashion from $\Psi$, then

(i) investors optimally choose $\Delta=\Delta_{t}$, thus incurring cost $F\left(\Delta_{t} ; \Delta_{t}\right)$;

(ii) the risk-free rate is constant over time and given as the unique positive solution to

$$
1=\beta(1+r) E\left[e^{\frac{\gamma^{2}}{2}\left(\frac{r}{1+r-\rho}\right)^{2}(1-\rho)^{2} \omega(\Delta)}\right]
$$

where the expectation is taken over the distribution of $\Delta$;

(iii) the risky-asset prices equal

$$
P_{t, j}\left(\Delta_{t}, D_{t, j}\right)=\phi\left(D_{t, j}-1\right)+\frac{1}{r}-\Phi_{1} \omega\left(\Delta_{t}\right)-\Phi_{0}
$$

with $\phi \equiv \frac{\rho}{1+r-\rho}$ and

$$
\begin{aligned}
\Phi_{1} & =\gamma \frac{r(1-\rho)^{2}}{(1+r-\rho)^{2}} \\
\Phi_{0} & =\frac{\Phi_{1}}{r} \frac{E\left[e^{\frac{\gamma^{2}}{2}\left(\frac{r}{1+r-\rho}\right)^{2}(1-\rho)^{2} \omega(\Delta)} \omega(\Delta)\right]}{E\left[e^{\frac{\gamma^{2}}{2}\left(\frac{r}{1+r-\rho}\right)^{2}(1-\rho)^{2} \omega(\Delta)}\right]}>0 .
\end{aligned}
$$

Furthermore, investors' optimal portfolios of risky assets are given by (12).

Equation (31) decomposes the price of a security into three components. As in all CARA models, one of these components equals the expected discounted value of future dividends, $\phi\left(D_{t, j}-1\right)+r^{-1}$. The other two capture the risk premium. The term $\Phi_{1} \omega\left(\Delta_{t}\right)$ is the risk premium associated with the realization of time- $t+1$ dividend uncertainty, to which each investor is exposed according to

${ }^{25}$ Alternatively, in the interest of simplicity, we could fix the interest exogenously to the model and let aggregate lending adjust accordingly. 
the breadth $\Delta_{t}$ of her time- $t$ portfolio. Finally, $\Phi_{0}$ equals the sum of the expected discounted value of risk premia due to future realizations of dividend innovations and $\Delta_{t}$.

We emphasize that the risk premium decreases with $\Delta_{t}$ and is common for all securities. Alternatively phrased, increases in capital movements across locations are correlated with higher prices for all risky securities (and hence lower expected excess returns). Importantly, these movements in the prices of risky securities are uncorrelated with movements in aggregate output or the interest rate, which are both constant by construction.

A further immediate implication of equation (31) is that the presence of repeated shocks to participation costs introduces correlation in security prices that exceeds that of their dividends. Indeed, taking two securities $j$ and $k$, and noting that $\operatorname{Var}\left(D_{t, j}\right)=\operatorname{Var}\left(D_{t, k}\right)$, we can use equation (31) to compute

$$
\operatorname{corr}\left(P_{t, j}, P_{t, i}\right)=\frac{\operatorname{Var}\left(\Phi_{1} \omega\left(\Delta_{t}\right)\right)+\phi^{2} \operatorname{cov}\left(D_{t, j}, D_{t, k}\right)}{\operatorname{Var}\left(\Phi_{1} \omega\left(\Delta_{t}\right)\right)+\phi^{2} \operatorname{Var}\left(D_{t, j}\right)}>\frac{\operatorname{cov}\left(D_{t, j}, D_{t, k}\right)}{\operatorname{Var}\left(D_{t, j}\right)}=\operatorname{corr}\left(D_{t, j}, D_{t, i}\right) .
$$

The intuition is that movements in market integration cause common movements in the pricing of risk which make prices more correlated (and volatile) than the underlying dividends.

We collect some basic properties of the price due to the randomness in $\Delta_{t}$ in the following proposition.

Proposition 6 (i) $P_{t, i}$ increases with $\Delta_{t}$, and therefore $\operatorname{corr}\left(P_{t, i}, \Delta_{t}\right)>0$;

(ii) $E_{t}\left[P_{i, t+1}-(1+r) P_{i, t}\right]$ decreases with $\Delta_{t}$;

(iii) $\operatorname{corr}\left(P_{t, i}, P_{t, j}\right)>\operatorname{corr}\left(D_{t, i}, D_{t, j}\right)$;

(iv) $\Phi_{0}$ is higher (and hence the unconditional expected price is lower) than the one obtaining for $\Delta_{t}$ constant and equal to $E[\Delta]$.

\section{A.2 Multiple arcs on the circle}

The baseline model assumes that investors participate in markets spanning a single arc of length $\Delta$ around their "home" location. Extending the results to the general case where investors can choose to participate on multiple, disconnected arcs (as illustrated on the right-most graph of Figure 4) is straightforward and involves essentially no new insights. In this section we briefly sketch how to extend the results of the baseline model to this case and we show that allowing for this extra generality introduces an additional source of non-concavity into an investor's optimization problem.

To start, we introduce the function

$$
\begin{aligned}
& v(I)=\min _{N_{i}, \vec{a}_{i}, \vec{\Delta}_{i}, G_{j}^{(i)}} \operatorname{Var}\left(\int_{0}^{1} D_{j} d G_{j}^{(i)}\right) \\
& \text { s.t. } \quad I \geq F\left(\vec{a}_{i}, \sum_{n=1}^{N_{i}} \Delta_{i, n}\right) .
\end{aligned}
$$

In words, the function $v(I)$ is the minimal variance, per share purchased, of the portfolio payoff that can be obtained by an investor who is willing to spend an amount $I$ on participation costs. Proceeding similarly to Section 4.2 under the assumption that $P_{j}=P$ for all $j$, the facts that $U$ is exponential and all $D_{j}$ are normally distributed imply that maximizing utility over the choice of 


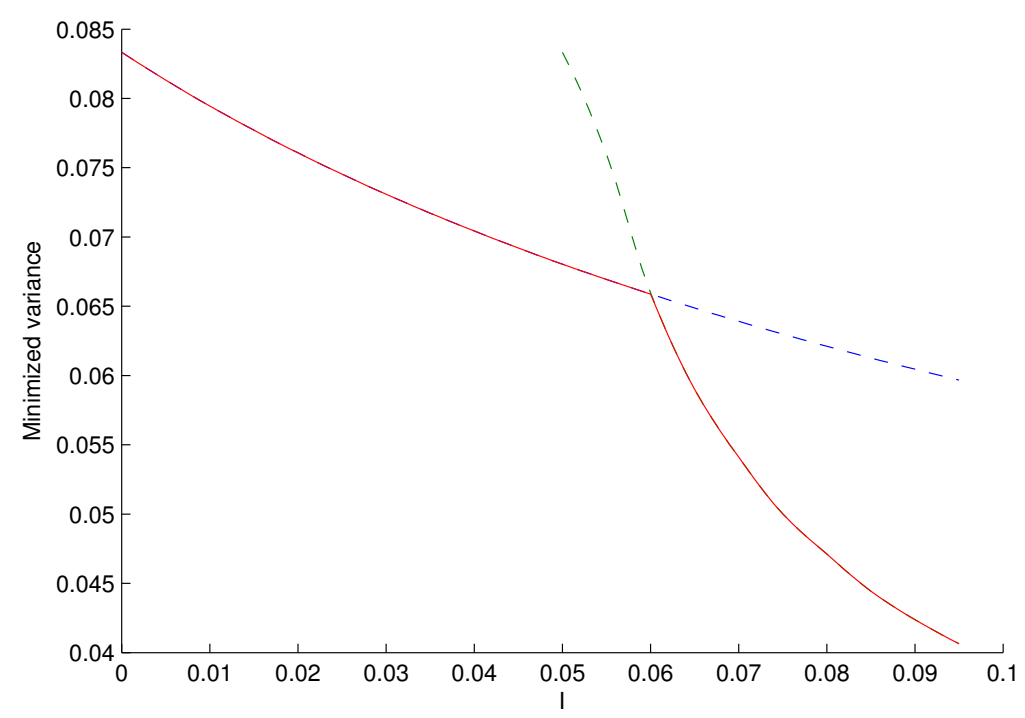

Figure 12: Numerical example to illustrate that $v(I)$ is non-convex. The figure depicts two (dotted) lines and the minimum of the two lines (solid line). The first dotted line starts at $I=0$ and depicts the minimal variance that can be attained when participation costs are equal to $I$ and the investor chooses to participate only on a single arc centered at her home location. The second dotted line starts at $I=0.05$, i.e. at the minimum expenditure required to invest in two distinct arcs. This second dotted line depicts the minimal variance that can be attained when participation costs are equal to $I$ and the investor can participate on two separate arcs with locations and lengths chosen so as to minimize variance. The function $v(I)$ (the minimum of the two dotted lines) is given by the solid line. For this example we chose $\sigma=1, g(x)=0.1 \times\left((1-x)^{-6}-1\right)$, $f(y)=0.05+0.005 \times\left(\left(\frac{1}{2}-y\right)^{-2}-\frac{1}{0.25}\right)$. (For $I>0.1$ the function $v(I)$ would in general exhibit further kinks at the critical values $I_{N}, N \geq 2$, where the investor is indifferent between $N$ and $N+1$ distinct arcs.)

$N_{i},\left\{a_{i, 1} ; . . ; a_{i, N_{i}}\right\},\left\{\Delta_{i, 1} ; . . ; \Delta_{i, N_{i}}\right\}, G_{j}^{(i)}$, and $w_{i}^{f}$ is equivalent to solving ${ }^{26}$

$$
\max _{w_{i}^{f}, I} P w_{i}^{f}+\left(1-w_{i}^{f}\right) \int_{0}^{1} E\left[D_{j}\right] d G_{j}^{(i)}-\frac{\gamma}{2}\left(1-w_{i}^{f}\right)^{2} v(I)-I .
$$

\footnotetext{
${ }^{26}$ To ensure that the optimization problem (34) has a solution it is convenient either to impose a collateral constraint such as (23) or to introduce some (potentially small but positive) aggregate risk in dividends. Either of these assumptions coupled with the additional assumption $\lim _{\Delta \rightarrow 1} g(\Delta)=\infty$ suffices to ensure the existence of a solution to (34). Alternatively, one can ensure that (34) has an interior solution by requiring that, upon plugging in the optimal value of $w_{i}^{f}$, the maximand in (35) tends to negative infinity as $I$ goes to infinity. Given the lower bound on $P$ provided by the autarky equilibrium, it suffices that $\lim _{I \rightarrow \infty}\left(\frac{\sigma^{2}}{12}\right)^{2} \frac{\gamma}{v(I)}-I=-\infty$. This condition may be harder to verify than Assumption 1 , since it is not readily expressed in terms of primitive parameters.
} 
Given that $E\left[D_{j}\right]=1$, equation (34) can be rewritten as

$$
V=\max _{I, w_{i}^{f}} P w_{i}^{f}+\left(1-w_{i}^{f}\right)-\frac{\gamma}{2}\left(1-w_{i}^{f}\right)^{2} v(I)-I .
$$

In the baseline version of the model (Section 4.2), where the investor chooses to invest in a single arc around her home location, $v$ is a convex function of the total cost $I{ }^{27}$ In the general case where investors' portfolios are invested on disconnected arcs, the function $v(I)$ is in general non-convex with kinks at the expenditure levels $I_{n}$ where it becomes optimal to invest in $n+1$ rather than $n$ distinct arcs. Figure 12 provides an illustration. This non-convexity of $v(I)$, which may arise when (and only when) investors participate in markets located on multiple distinct arcs, constitutes an additional reason for the maximization problem (35) to be non-concave. This reason is distinct from the non-concavity arising from the interaction between leverage and participation decisions that we identify in Section 4.3, and strengthens the conclusion that a symmetric equilibrium may not exist.

\section{B Proofs}

Proof of Lemma 1. Property 2 follows immediately from integrating (3). To show property 3, note that, for any $i \in(0,1), \lim _{d(i, j) \rightarrow 0} D_{j}=\lim _{j \rightarrow i} D_{j}=D_{i}$ a.s. by the continuity of the Brownian motion. Continuity at 0 follows from the fact that $B_{0}=B_{1}$.

We turn now to property 1 . Since $E\left(B_{i}\right)=0$ for all $i \in[0,1], E\left(D_{j}\right)=1$. To compute $\operatorname{cov}\left(D_{i}, D_{j}\right)$ we start by noting that $\operatorname{cov}\left(B_{s}, B_{t}\right)=E\left(B_{s} B_{t}\right)=s(1-t)$ for $s \leq t$. Therefore, for any $t \in[0,1]$

$$
\begin{aligned}
\int_{0}^{1} E\left(B_{t} B_{u}\right) d u & =\int_{0}^{t} u(1-t) d u+\int_{t}^{1} t(1-u) d u \\
& =\frac{1}{2}(1-t) t^{2}+\frac{1}{2}(1-t)^{2} t=\frac{t(1-t)}{2} .
\end{aligned}
$$

Accordingly,

$$
\begin{aligned}
\operatorname{Var}\left(\int_{0}^{1} B_{u} d u\right) & =E\left[\left(\int_{0}^{1} B_{u} d u\right)^{2}\right]=E\left[\left(\int_{0}^{1} B_{u} d u\right)\left(\int_{0}^{1} B_{t} d t\right)\right] \\
& =\int_{0}^{1}\left(\int_{0}^{1} E\left(B_{u} B_{t}\right) d u\right) d t=\int_{0}^{1} \frac{t(1-t)}{2} d t=\frac{1}{12}
\end{aligned}
$$

where the second line of (37) follows from Fubini's Theorem and (36). Combining (37) and (36)

\footnotetext{
${ }^{27}$ To see this, note that $v^{\prime}(I)=\frac{\omega^{\prime}(\Delta)}{\kappa g^{\prime}(\Delta)}$, where $\Delta(I)=g^{-1}\left(\frac{I}{\kappa}\right)$. Differentiating again gives $v^{\prime \prime}(I)=$ $\frac{1}{\kappa} \frac{\omega^{\prime \prime}(\Delta) g^{\prime}(\Delta)-\omega^{\prime}(\Delta) g^{\prime \prime}(\Delta)}{\left(g^{\prime}(\Delta)\right)^{2}} \Delta^{\prime}(I)>0$.
} 
gives

$$
\begin{aligned}
\frac{1}{\sigma^{2}} \operatorname{Var}\left(D_{t}\right) & =\operatorname{Var}\left(B_{t}\right)+\operatorname{Var}\left(\int_{0}^{1} B_{u} d u\right)-2 \operatorname{cov}\left(B_{t}, \int_{0}^{1} B_{u} d u\right) \\
& =t(1-t)+\frac{1}{12}-2 \int_{0}^{1} E\left(B_{t} B_{u}\right) d u=\frac{1}{12} .
\end{aligned}
$$

This calculation finishes the proof of property 1. For property 4, take any $s \leq t$ and use (36) and (37) to obtain

$$
\begin{aligned}
\frac{\operatorname{cov}\left(D_{s}, D_{t}\right)}{\sigma^{2}} & =\operatorname{cov}\left(B_{s}-\int_{0}^{1} B_{u} d u, B_{t}-\int_{0}^{1} B_{u} d u\right) \\
& =E\left(B_{s} B_{t}\right)-E\left(B_{s} \int_{0}^{1} B_{u}\right) d u-E\left(B_{t} \int_{0}^{1} B_{u}\right) d u+\frac{1}{12} \\
& =s(1-t)-\frac{s(1-s)}{2}-\frac{t(1-t)}{2}+\frac{1}{12} \\
& =\frac{(s-t)(1+s-t)}{2}+\frac{1}{12} .
\end{aligned}
$$

This establishes property 4.

Proof of Proposition 1. We start by establishing the following lemma.

Lemma 2 The (bounded-variation) function $L$ with $L_{-\frac{\Delta}{2}^{-}}=0$ and $L_{\frac{\Delta}{2}}=1$ that minimizes $\operatorname{Var}\left(\int_{-\frac{\Delta}{2}^{-}}^{\frac{\Delta}{2}} D_{j} d L_{j}\right)$ is given by (12). Moreover, the minimal variance is equal to $\omega(\Delta)$.

Proof of Lemma 2. To simplify notation, we prove a "shifted" version of the lemma, namely finding the minimal-variance portfolio on $[0, \Delta]$ rather than $\left[-\frac{\Delta}{2}, \frac{\Delta}{2}\right]$. The two versions are clearly equivalent, since covariances depend only on the distances between locations, rather than the locations themselves.

We start by defining $q(d)=\frac{1}{12}-\frac{d(1-d)}{2}$ and therefore $q^{\prime}(d)=-\frac{1}{2}+d$. In light of $(4), q(d)=$ $\frac{1}{\sigma^{2}} \operatorname{cov}\left(D_{i}, D_{j}\right)$ whenever $d(i, j)=d$. If $L_{u}=\int_{0^{-}}^{u} d L_{u}$ is a variance-minimizing portfolio of risky assets, it must be the case that the covariance between any gross return $R_{s}=\frac{D_{s}}{P}$ for $s \in[0, \Delta]$ and the portfolio $\int_{0^{-}}^{\Delta} R_{u} d L_{u}=\int_{0^{-}}^{\Delta} \frac{D_{u}}{P} d L_{u}$ is independent of $s$. Thus, the quantity

$$
\begin{aligned}
\frac{1}{\sigma^{2}} \operatorname{cov}\left(D_{s}, \int_{0^{-}}^{\Delta} D_{u} d L_{u}\right) & =\frac{1}{\sigma^{2}}\left[\int_{0^{-}}^{s} \operatorname{cov}\left(D_{s}, D_{u}\right) d L_{u}+\int_{s}^{\Delta} \operatorname{cov}\left(D_{s}, D_{u}\right) d L_{u}\right]= \\
& =\int_{0^{-}}^{s} q(s-u) d L_{u}+\int_{s}^{\Delta} q(u-s) d L_{u}
\end{aligned}
$$

is independent of $s$. Letting $\tilde{L}(s)=1-L(s)$ and integrating by parts we obtain

$$
\begin{aligned}
& \int_{0^{-}}^{s} q(s-u) d L_{u}=L(s) q(0)-L\left(0^{-}\right) q(s)+\int_{0^{-}}^{s} L_{u} q^{\prime}(s-u) d u \\
& \int_{s}^{\Delta} q(u-s) d L_{u}=\tilde{L}(s) q(0)-\tilde{L}(\Delta) q(\Delta-s)+\int_{s}^{\Delta} \tilde{L}_{u} q^{\prime}(u-s) d u .
\end{aligned}
$$


Using (41) and (42) inside (40) and recognizing that $q(0)=\frac{1}{12}, L\left(0^{-}\right)=0$, and $\tilde{L}(\Delta)=0$, we obtain that (40) equals

$$
Q(s) \equiv \frac{1}{12}+\int_{0^{-}}^{s} L_{u} q^{\prime}(s-u) d u+\int_{s}^{\Delta} \tilde{L}_{u} q^{\prime}(u-s) d u
$$

This expression is independent of $s \in[0, \Delta]$ if and only if $Q^{\prime}(s)=0$. Differentiating (43) and setting the resulting expression to zero yields

$$
\begin{aligned}
Q^{\prime}(s) & =\int_{0^{-}}^{s} L_{u} q^{\prime \prime}(s-u) d u-\int_{s}^{\Delta} \tilde{L}_{u} q^{\prime \prime}(u-s) d u+L_{s} q^{\prime}(0)-\tilde{L}_{s} q^{\prime}(0) \\
& =\int_{0^{-}}^{\Delta} L_{u} d u-\Delta+s-L_{s}+\frac{1}{2}=0
\end{aligned}
$$

where we used $q^{\prime}(0)=-\frac{1}{2}, q^{\prime \prime}=1$, and $\tilde{L}(s)=1-L(s)$. Since (44) needs to hold for all $s \in[0, \Delta]$, it must be the case that $L_{s}=A+s$ for an appropriate constant $A$. To determine $A$, we subsitute $L_{s}=A+s$ into (44) and solve for $A$ to obtain

$$
A=\frac{1-\Delta}{2} \text {. }
$$

It is immediate that the standardized portfolio corresponding to the solution $L$ we computed is $L^{*}$ of $(12)$.

Using the variance-minimizing portfolio inside (43), implies after several simplifications, that $Q=\frac{1}{12}(1-\Delta)^{3}$ and hence $\operatorname{cov}\left(D_{s}, \int_{0^{-}}^{\Delta} D_{u} d L_{u}\right)=Q \sigma^{2}=\omega(\Delta)$. Accordingly,

$$
\begin{aligned}
\operatorname{Var}\left(\int_{0^{-}}^{\Delta} D_{u} d L_{u}\right) & =\operatorname{cov}\left(\int_{0^{-}}^{\Delta} D_{s} d L_{s}, \int_{0^{-}}^{\Delta} D_{u} d L_{u}\right)=\int_{0^{-}}^{\Delta} \operatorname{cov}\left(D_{s}, \int_{0^{-}}^{\Delta} D_{u} d L_{u}\right) d L_{s} \\
& =\omega(\Delta) \int_{0^{-}}^{\Delta} d L_{s}=\omega(\Delta) .
\end{aligned}
$$

With Lemma 2 in hand it is possible to confirm that the allocations and prices of Proposition 1 constitute a symmetric equilibrium - assuming that one exists. We already argued that all agents choose the same standardized portfolio (as agent $\frac{\Delta}{2}$ ). Furthermore, since in a symmetric equilibrium all agents must hold the same allocation of bonds, clearing of the bond market requires $w_{i}^{f}=0$ for all $i$. By equation (18), $w_{i}^{f}=0$ is supported as an optimal choice for an investor only if $P_{i}=P$ is given by (15). Similarly, in light of (19), equation (14) is a necessary optimality condition for the interval $\Delta^{*}$. Since the values of $P$ and $\Delta^{*}$ implied by (15) and (14) are unique, they are necessarily the equilibrium values of $P$ and $\Delta^{*}$ that characterize a symmetric equilibrium. Hence, when a symmetric equilibrium exists, it is unique in the class of symmetric equilibria.

Existence of a symmetric equilibrium implies that $w_{i}^{f}=0$ is optimal, and so are the choices $\Delta^{*}$ and $G_{i+j}^{(i)}=L_{j}^{*}$ given prices $P_{i}=P$. It remains to show that markets clear. We already addressed bond-market clearing. To see that the stock markets clear, we start by noting that, since $P_{i}=W_{0, i}=P$ for all $i$, the market clearing condition amounts to $\int_{i \in[0,1)} d G_{j}^{(i)}=1$. We have $\int_{i \in[0,1)} d G_{j}^{(i)}=\int_{i \in[0,1)} d L_{j-i}^{*}=\int_{j \in[0,1)} d L_{j}^{*}=1$. 
Proof of Proposition 2. Let $w^{*}(P)$ denote the set of optimal $w_{i}^{f}$ solving the maximization problem (17) when the price in all markets is $P$. We first note that the assumption that no symmetric equilibrium exists implies that there exists no $P$ such that $0 \in w^{*}(P)$. (If such a $P$ existed, then we could simply repeat the arguments of Proposition 1 to establish the existence of a symmetric equilibrium with price $P_{i}=P$, and interval choice $\left.\Delta_{i}=\Delta^{*}(P)\right)$.

We next show that since there exists no $P$ such that $0 \in w^{*}(P)$, it follows that $w^{*}(P)$ cannot be single-valued for all $P$. We argue by contradiction. Suppose to the contrary that $w^{*}(P)$ is single-valued. Since the theorem of the maximum implies that $w^{*}(P)$ is a upper-hemicontinuous correspondence, it follows that $w^{*}(P)$ is actually a continuous function. Inspection of (17) shows that $w^{*}(1)=1$. Moreover, as $P \rightarrow-\infty$, the optimal solution to (17) becomes negative: $w^{*}(-\infty)<$ 0 . Then an application of the intermediate value theorem gives the existence of $P$ such that $w^{*}(P)=0$, a contradiction.

Combining the facts that a) there exists no $P$ such that $\left.0 \in w^{*}(P), \mathrm{b}\right) w^{*}(P)$ is multi-valued for at least one value of $P$, and c) $w^{*}(P)$ is upper-hemicontinuous, implies that there exists at least one $P$ such that $\left\{w_{1}, w_{2}\right\} \in w^{*}(P)$ with $w_{1}>0$ and $w_{2}<0$. An implication of the necessary first-order condition for the optimality of the interval choice $\Delta^{*}(P)$ is that $\Delta^{*}(P)$ is also multi-valued with $\Delta_{1}<\Delta_{2}$. Furthermore, since prices in all locations are equal, the (standardized) optimal portfolio of an agent choosing $\Delta_{k}$ is the variance-minimizing portfolio of Proposition 1 , denoted $L^{*, k}$.

From this point onwards, an equilibrium can be constructed as follows. By definition, the tuples $\left\{\Delta_{1}, w_{1}, d L^{*, 1}\right\}$ and $\left\{\Delta_{2}, w_{2}, d L^{*, 2}\right\}$ are optimal. Hence it only remains to confirm that asset markets clear. Define $\pi \equiv-\frac{w_{2}}{w_{1}-w_{2}} \in(0,1)$. By construction, $\pi w_{1}+(1-\pi) w_{2}=0$ and, therefore, if in every location $\pi$ agents choose $\left\{\Delta_{1}, w_{1}, d L^{*, 1}\right\}$ and the remaining fraction $(1-\pi)$ choose $\left\{\Delta_{2}, w_{2}, d L^{*, 2}\right\}$, then the bond market clears by construction. To see that the stock markets clear, we start by noting that, since $P_{i}=W_{0, i}=P$, the market clearing condition for stock $i$ amounts to

$$
\pi \int_{[0,1]} d L_{j-i}^{*, 1}+(1-\pi) \int_{[0,1]} d L_{j-i}^{*, 2}=1
$$

which holds because $L_{j}^{*, k}$ for $k \in\{1,2\}$ is a measure on the circle.

We prove next that symmetric and asymmetric equilibria (with different prices) cannot coexist. Inspection of (17) shows that the optimal $w_{i}^{f}$ is increasing in $P$ in the sense that if $P_{1}<P_{2}$ then $w_{1}<w_{2}$ for any $w_{1} \in w^{*}\left(P_{1}\right)$ and $w_{2} \in w^{*}\left(P_{2}\right){ }^{28}$ Accordingly, if a symmetric equilibrium exists, i.e., if there is a $\hat{P}$ such that $0 \in w^{*}(\hat{P})$ then there cannot exist $P \neq \hat{P}$ with the property that $\left\{w_{1}, w_{2}\right\} \in w(P)$ and yet $w_{1}>0$ and $w_{2}<0$, which is a requirement for the existence of a symmetric equilibrium. Hence symmetric and asymmetric equilibria cannot co-exist. The fact that $w^{*}(P)$ is an increasing correspondence also implies that asymmetric equilibria are essentially unique, in the sense that we cannot have the co-existence of asymmetric equilibria associated with different equilibrium prices.

Remark 1 The existence proof of an asymmetric equilibrium (when a symmetric equilibrium fails to exist) obtains also in the presence of the leverage constraint (23) that we introduce in Section 5.

Proof of Proposition 3. Conjecture first that in equilibrium $P_{j}=P$ for all $j$ and let $\pi \in[0,1]$ denote the fraction of funds invested in the local market. Assuming that a given investor

${ }^{28}$ To see that this statement is correct, consider the maximum $\bar{V}\left(P, w_{i}^{f}\right)$ of the maximand in (17) over $\Delta$ and note that it cross-partial derivative with respect to $P$ and $w_{i}^{f}$ is positive: $\partial_{P} \partial_{w_{i}^{f}} \bar{V}\left(P, w_{i}^{f}\right)>0$. 
chooses $N=2$ (that is, chooses to invest in her own location and another location at distance $d$ ), equation (4) allows the computation of the minimal portfolio variance:

$$
\begin{aligned}
\widehat{\omega}(d) & =\sigma^{2} \min _{\pi}\left\{\left(\pi^{2}+(1-\pi)^{2}\right) \frac{1}{12}+2 \pi(1-\pi)\left(\frac{1}{12}-\frac{d(1-d)}{2}\right)\right\} \\
& =\sigma^{2}\left(\frac{1}{12}-\frac{1}{4} d(1-d)\right) .
\end{aligned}
$$

The optimal distance $d$ for an investor choosing $N=2$ satisfies a first-order condition similar to equation (19), namely

$$
-\frac{\gamma}{2}\left(1-w^{f}\right)^{2} \frac{\widehat{\omega}^{\prime}(d)}{\widehat{\omega}^{2}(d)}=\kappa f^{\prime}(d) .
$$

Since $\widehat{\omega}^{\prime}(d)=0$ when and only when $d=\frac{1}{2}$, and $f^{\prime}(d)=0$, it follows that $d=\frac{1}{2}$ is optimal for an investor choosing $N=2$. Hence the minimal portfolio variance of an investor choosing $N=1$ is equal to $\widehat{\omega}(0)=\frac{\sigma^{2}}{12}$, while the minimal portfolio variance for an investor choosing $N=2$ is $\widehat{\omega}\left(\frac{1}{2}\right)=\frac{\sigma^{2}}{48}$. Assuming that the equilibrium is of the asymmetric, location-invariant type, we can use equation (17) to express the indifference between the choices $N=1$, respectively $N=2$ and $d=\frac{1}{2}$, as

$$
P w_{1}^{f}+\left(1-w_{1}^{f}\right)-\frac{\gamma}{2}\left(1-w_{1}^{f}\right)^{2} \widehat{\omega}(0)=P w_{2}^{f}+\left(1-w_{2}^{f}\right)-\frac{\gamma}{2}\left(1-w_{2}^{f}\right)^{2} \widehat{\omega}\left(\frac{1}{2}\right)-\kappa f_{0} .
$$

Using the first-order conditions for leverage

$$
1-P=\gamma\left(1-w_{2}^{f}\right) \widehat{\omega}\left(\frac{1}{2}\right)=\gamma\left(1-w_{1}^{f}\right) \widehat{\omega}(0)
$$

inside (47) yields - after some simplifications - the equilibrium price (24).

To verify that the postulated equilibrium is indeed an equilibrium, we proceed as in the proof of Proposition 2. For $P(\kappa)$ to be an equilibrium price in all locations, it must also be case that $1-w_{1}^{f} \leq 1 \leq 1-w_{2}^{f}$, so that setting $\pi=\frac{w_{2}^{f}}{w_{2}^{f}-w_{1}^{f}}>0$ ensures market clearing (of bond markets and all risky asset markets). In light of (48), the requirement $1-w_{1}^{f} \leq 1 \leq 1-w_{2}^{f}$ is equivalent to $P \in\left[1-\gamma \widehat{\omega}(0), 1-\gamma \widehat{\omega}\left(\frac{1}{2}\right)\right]$. This requirement is satisfied as long as $\kappa \in\left(\kappa_{1}, \kappa_{2}\right)$.

Lemma 3 Consider an investor located at $i \notin\left[-\frac{k}{2} ; \frac{k}{2}\right]$, and therefore investing in markets $[i-$ $\left.\frac{\bar{\Delta}}{2}, i+\frac{\bar{\Delta}}{2}\right]$. Suppose that $P(x)$ is continuously differentiable everywhere on $\left[i-\frac{\bar{\Delta}}{2}, i+\frac{\bar{\Delta}}{2}\right]$. With $d X_{l}^{(i)}$ the number of shares purchased on the account of an investor at $i$ in market $l$ and $j \equiv i-\frac{\bar{\Delta}}{2}$,

$$
X_{j+\bar{\Delta}}^{(i)}=\frac{1}{\gamma \omega(\bar{\Delta})}\left[1-\frac{1-\bar{\Delta}}{2}\left(P_{j}+P_{j+\bar{\Delta}}\right)-\int_{j}^{j+\bar{\Delta}} P_{u} d u\right] .
$$


Furthermore, the function $X$ is given by

$$
X_{j+l}^{(i)}=\frac{P_{j+l}^{\prime}}{\gamma \sigma^{2}}+X_{j+\bar{\Delta}}^{(i)} \frac{1-\bar{\Delta}+2 l}{2}+\frac{1}{1-\bar{\Delta}} \frac{P_{j+\bar{\Delta}}-P_{j}}{\gamma \sigma^{2}} .
$$

If an investor is located at $i \in\left[-\frac{k}{2}, \frac{k}{2}\right]$ and only invests in market $i$ then the respective demand for risky asset $i$ is given by

$$
\widehat{X}_{i}^{(i)}=\frac{1}{\gamma \omega(0)}\left(1-P_{i}\right) .
$$

Proof of Lemma 3. Notice that optimization problem of agent $i$ is equivalent to

$$
\max _{X} P_{i}+\int_{j^{-}}^{j+\bar{\Delta}}\left(1-P_{u}\right) d X_{u}-\frac{\gamma}{2} \operatorname{Var}\left(\int_{j^{-}}^{j+\bar{\Delta}} D_{u} d X_{u}\right)
$$

Thus, the first-order condition requires that

$$
\gamma \operatorname{cov}\left(D_{s}, \int_{j^{-}}^{j+\bar{\Delta}} D_{u} d X_{u}\right)=1-P_{s}
$$

for all $s \in[j, j+\bar{\Delta}]$. Letting $q(d)$ be defined as in Lemma 2 we can rewrite (53) as

$$
\int_{j^{-}}^{s} q(s-u) d X_{u}+\int_{s}^{j+\Delta} q(u-s) d X_{u}=\frac{1-P_{s}}{\gamma \sigma^{2}} .
$$

Let $\widetilde{X}(s)=X(j+\bar{\Delta})-X(s)$ and integrating by parts we obtain

$$
\begin{aligned}
\int_{j^{-}}^{s} q(s-u) d X_{u} & =X(s) q(0)-X\left(j^{-}\right) q(s)+\int_{j}^{s} X_{u} q^{\prime}(s-u) d u \\
\int_{s}^{j+\Delta} q(u-s) d X_{u} & =\widetilde{X}(s) q(0)-\widetilde{X}(\bar{\Delta}) q(\Delta-s)+\int_{s}^{j+\bar{\Delta}} \widetilde{X}_{u} q^{\prime}(u-s) d u
\end{aligned}
$$

Substituting (55) and (56) into (54), recognizing that $q(0)=\frac{1}{12}, X\left(j^{-}\right)=0$, and $\tilde{X}(j+\bar{\Delta})=0$, we obtain

$$
\frac{1}{12} X(j+\bar{\Delta})+\int_{j}^{s} X_{u} q^{\prime}(s-u) d u+\int_{s}^{j+\bar{\Delta}} \widetilde{X}_{u} q^{\prime}(u-s) d u=\frac{1-P_{s}}{\gamma \sigma^{2}} .
$$

Since this relation must hold for all $s$, we may differentiate both sides of (57) to obtain

$$
\int_{j}^{s} X_{u} q^{\prime \prime}(s-u) d u-\int_{s}^{j+\bar{\Delta}} \widetilde{X}_{u} q^{\prime \prime}(u-s) d u+X_{s} q^{\prime}(0)-\widetilde{X}_{s} q^{\prime}(0)=-\frac{P_{s}^{\prime}}{\gamma \sigma^{2}} .
$$

This equation holds for all $s \in(j, j+\bar{\Delta})$. Noting that $q^{\prime \prime}=1, q^{\prime}(0)=-\frac{1}{2}, \widetilde{X}(s)=X(j+\bar{\Delta})-$ 
$X(s)$, and using (58) to solve for $X_{s}$ yields

$$
X_{s}=\int_{j}^{j+\bar{\Delta}} X_{u} d u+\left(s-j+\frac{1}{2}-\bar{\Delta}\right) X(j+\bar{\Delta})+\frac{P_{s}^{\prime}}{\gamma \sigma^{2}} .
$$

Integrating (59) from $j$ to $j+\bar{\Delta}$ and solving for $\int_{j}^{j+\bar{\Delta}} X_{u} d u$ leads to

$$
\int_{j}^{j+\bar{\Delta}} X_{u} d u=\frac{1}{1-\bar{\Delta}}\left[X(j+\bar{\Delta}) \bar{\Delta}\left(\frac{1-\bar{\Delta}}{2}\right)+\frac{P(j+\bar{\Delta})-P(j)}{\gamma \sigma^{2}}\right],
$$

so that

$$
\begin{aligned}
X_{s}= & \frac{1}{1-\bar{\Delta}}\left[X(j+\bar{\Delta}) \bar{\Delta}\left(\frac{1-\bar{\Delta}}{2}\right)+\frac{P(j+\bar{\Delta})-P(j)}{\gamma \sigma^{2}}\right]+ \\
& \left(s-j+\frac{1}{2}-\bar{\Delta}\right) X(j+\bar{\Delta})+\frac{P_{s}^{\prime}}{\gamma \sigma^{2}} .
\end{aligned}
$$

Evaluating (57) at $s=j+\bar{\Delta}$, and noting that $q^{\prime}(s)=-\frac{1}{2}+s$ leads to

$$
\frac{1}{12} X(j+\bar{\Delta})+\int_{j}^{j+\bar{\Delta}} X_{u}\left[-\frac{1}{2}+(\bar{\Delta}-u)\right] d u=\frac{1-P_{j+\bar{\Delta}}}{\gamma \sigma^{2}} .
$$

An implication of (59) is that $X_{u}=X_{j}+\frac{P_{u}^{\prime}-P_{j}^{\prime}}{\gamma \sigma^{2}}+X(j+\bar{\Delta}) u$. Using this expression for $X_{u}$ inside (62), carrying out the requisite integrations and using integration by parts to express $\int_{j}^{j+\bar{\Delta}}\left(\frac{P_{u}^{\prime}}{\gamma \sigma^{2}}\right) u d u=\frac{P_{j+\bar{\Delta}}}{\gamma \sigma^{2}}(j+\bar{\Delta})-\frac{P_{j}}{\gamma \sigma^{2}} j-\int_{j}^{j+\bar{\Delta}} \frac{P_{u}}{\gamma \sigma^{2}} d u$, leads (after some simplifications) to

$$
\begin{aligned}
X(j+ & \bar{\Delta})\left(\frac{1}{12}+\frac{\bar{\Delta}^{3}}{6}-\frac{\bar{\Delta}^{2}}{4}\right)-\frac{\bar{\Delta}(1-\bar{\Delta})}{2}\left(X_{j}-\frac{P_{j}^{\prime}}{\gamma \sigma^{2}}\right)-\frac{P_{j+\bar{\Delta}}-P_{j}}{2 \gamma \sigma^{2}}+\int_{j^{-}}^{j+\bar{\Delta}} \frac{P_{u}-P_{j}}{\gamma \sigma^{2}} d u \\
& =\frac{1-P_{\bar{\Delta}}}{\gamma \sigma^{2}} .
\end{aligned}
$$

Finally, evaluating (59) at $j$ gives

$$
\left(X_{j}-\frac{P_{j}^{\prime}}{\gamma \sigma^{2}}\right)=\int_{j}^{j+\bar{\Delta}} X_{u} d u+\left(\frac{1}{2}-\bar{\Delta}\right) X(j+\bar{\Delta}) .
$$

Equations (60), (63), and (64) are three linear equations in three unknowns. Solving for $X(j+\bar{\Delta})$ and using the definition of $\omega(\bar{\Delta})$ leads to (49). Equation (61) simplifies to (50). Finally, (51) is a direct consequence of (53) when $\bar{\Delta}=0$. 
Proof of Proposition 4. For any $j \in\left(\frac{k}{2}, \frac{1}{2}\right]$ and $l \in\left(-\frac{\bar{\Delta}}{2}, \frac{\bar{\Delta}}{2}\right)$, we have from Lemma 3 :

$$
\begin{aligned}
X_{j-\frac{\bar{\Delta}}{2}}^{(j)} & =\frac{P_{j-\frac{\bar{\Delta}}{2}}^{\prime}}{\gamma \sigma^{2}}+\frac{P_{j-\frac{\bar{\Delta}}{2}}-P_{j+\frac{\bar{\Delta}}{2}}}{\gamma \sigma^{2}(1-\Delta)}+\frac{1-\Delta}{2} X_{j+\frac{\bar{\Delta}}{2}}^{(j)} \\
d X_{j+l}^{(j)} & =\left(\frac{P_{j+l}^{\prime \prime}}{\gamma \sigma^{2}}+X_{j+\frac{\bar{\Delta}}{2}}^{(j)}\right) d l \\
X_{j+\frac{\bar{\Delta}}{2}}^{(j)}-X_{\left(j+\frac{\bar{\Delta}}{2}\right)^{-}}^{(j)} & =-\frac{P_{j+\frac{\bar{\Delta}}{2}}^{\prime}}{\gamma \sigma^{2}}-\frac{P_{j-\frac{\bar{\Delta}}{2}}-P_{j+\frac{\bar{\Delta}}{2}}}{\gamma \sigma^{2}(1-\Delta)}+\frac{1-\Delta}{2} X_{j+\frac{\bar{\Delta}}{2}}^{(j)} .
\end{aligned}
$$

Specialize the first equation to $j=\frac{1}{2}+\frac{\Delta}{2}$, the second to $j=\frac{1}{2}-l$ for all $l \in\left(-\frac{\bar{\Delta}}{2}, \frac{\bar{\Delta}}{2}\right)$, and the third to $j=\frac{1}{2}-\frac{\Delta}{2}$ and aggregate to obtain the total demand for asset $\frac{1}{2}$ :

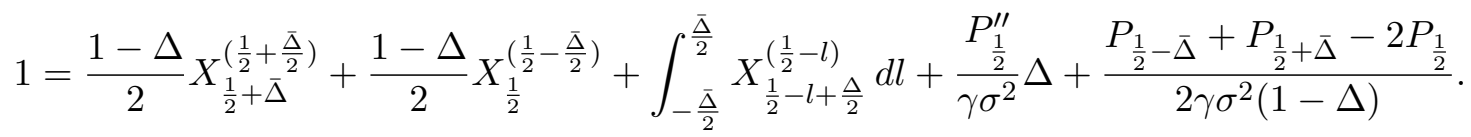

Suppose now that $P_{j} \geq 1-\gamma \omega(\bar{\Delta})$ on $\left[\frac{1}{2}-\Delta, \frac{1}{2}+\Delta\right]$, with strict inequality on a positive measure set. It then follows from equation (49) that $X_{j+\frac{\Delta}{2}}^{(j)} \leq 1$, so that

$$
0<\frac{P_{\frac{1}{2}}^{\prime \prime}}{\gamma \sigma^{2}} \Delta+\frac{P_{\frac{1}{2}-\bar{\Delta}}+P_{\frac{1}{2}+\bar{\Delta}}-2 P_{\frac{1}{2}}}{2 \gamma \sigma^{2}(1-\Delta)} .
$$

This inequality contradicts the assumption that $P$ is maximized at $\frac{1}{2}$.

Proof of Proposition 5. We adopt a guess-and-verify approach. We start by noting that the beginning-of-period wealth of investor $i$ at time $t+1$ is $W_{t+1, i} \equiv \int_{0}^{1}\left(P_{t+1, j}+D_{t+1, j}\right) d X_{t, j}^{(i)}+X_{B, t}^{(i)}$. We then conjecture that, as long as

$$
F(\Delta)=\frac{M}{\gamma}+\frac{\gamma}{2}\left(\frac{r}{1+r-\rho}\right)^{2}(1-\rho)^{2} \omega\left(\Delta_{t}\right)
$$

for some $M>-\frac{\gamma^{2}}{2}\left(\frac{r}{1+r-\rho}\right)^{2}(1-\rho)^{2} \omega\left(\Delta_{u}\right)$, (ii) and (iii) obtain. We show at the end that the function $F$ can be chosen to ensure (i).

We also conjecture and verify that investors' holdings of risky assets $X_{t, j}^{(i)}$ coincide with $G_{t, j}^{(i)}$ of Proposition 1, and that their bond holdings equal

$$
X_{B, t}^{(i)}=W_{t, i}-(1+r) \bar{P}_{t, i}-r \Phi_{t},
$$

where $\bar{P}_{t, i} \equiv \int_{0}^{1} P_{t, j} d X_{t, j}^{(i)}$ is the average price that investor $i$ pays for her portfolio. Here, to simplify notation, we defined $\Phi_{t} \equiv \Phi_{1} \omega\left(\Delta_{t}\right)+\Phi_{0}$.

We first ensure that with these postulates markets clear. Clearly, all risky markets clear, since the holdings of risky assets are the same as in Proposition 1. To show that bond markets clear, we proceed inductively. First we note that investors are endowed with no bonds at time 
zero. Hence $\int_{0}^{1} X_{B,-1}^{(i)} d i=0$ and therefore $\int_{0}^{1} W_{0, i} d i=\int_{0}^{1}\left(P_{0, i}+D_{0, i}\right) d i$. Next we postulate that $\int_{0}^{1} X_{B, t-1}^{(i)} d i=0$, so that $\int_{0}^{1} W_{t, i} d i=\int_{0}^{1} P_{t, i} d i+\int_{0}^{1} D_{t, j} d j$. Integrating our postulate (66) for $X_{B, t}^{(i)}$ across all investors, we obtain

$$
\int_{0}^{1} X_{B, t}^{(i)} d i=\int_{0}^{1} W_{t, i} d i-(1+r) \int_{0}^{1} \bar{P}_{t, i} d i-r \Phi_{t} .
$$

We next note that that (a) $\int_{0}^{1} D_{t, j} d j=1$, by construction of the dividend process; (b) $\int_{0}^{1} W_{t, i} d i=$ $\int_{0}^{1} P_{t, i} d i+\int_{0}^{1} D_{t, j} d j=r^{-1}-\Phi_{t}+1$, using the induction hypothesis, (31), and (a); and (c) $\int_{0}^{1} \bar{P}_{t, i} d i=$ $\int_{0}^{1} P_{t, i} d i=r^{-1}-\Phi_{t}$. Using these three facts, it follows immediately that the right-hand side of (67) is zero, so that the bond market clears.

If investors set their bond holdings according to (66), then their budget constraint implies a consumption of

$$
c_{t, i}=W_{t, i}-\frac{1}{1+r} X_{B, t}^{(i)}-\bar{P}_{t, i}-F_{t} .
$$

Using the definition of $W_{t, i}$ and market clearing condition for bond holdings inside (68), and integrating across $i$ implies that the market for consumption goods clears: $\int_{0}^{1} c_{t, i} d i=1-F_{t}$.

Having established market clearing given the postulated policies and prices, we next turn to optimality. Equation (68) implies

$$
\begin{aligned}
c_{t+1, i}-c_{t, i} & =W_{t+1, i}-W_{t, i}-\frac{1}{1+r}\left(X_{B, t+1}^{(i)}-X_{B, t}^{(i)}\right)-\left(\bar{P}_{t+1, i}-\bar{P}_{t, i}\right)-\left(F_{t+1}-F_{t}\right) \\
& =\left(\frac{r}{1+r}\right)\left(W_{t+1, i}-W_{t, i}\right)+\frac{r}{1+r}\left(\Phi_{t+1}-\Phi_{t}\right)-\left(F_{t+1}-F_{t}\right),
\end{aligned}
$$

where the second line follows from (66). We next use the definition of $W_{t, i}$ and (66) to obtain

$$
\begin{aligned}
W_{t+1, i}-W_{t, i} & =\int_{0}^{1}\left(P_{t+1, j}+D_{t+1, j}\right) d X_{t, j}^{(i)}+X_{B, t}^{(i)}-W_{t, i} \\
& =\int_{0}^{1}\left(P_{t+1, j}+D_{t+1, j}\right) d X_{t, j}^{(i)}-(1+r) \bar{P}_{t, i}-r \Phi_{t} .
\end{aligned}
$$

Substituting (70) into (69) and using (31) and (66) leads to

$$
\begin{aligned}
c_{t+1, i}-c_{t, i}= & \left(\frac{r}{1+r}\right)\left[(1+\phi) \int_{0}^{1} D_{t+1, j} d X_{t, j}^{(i)}-(1+r) \phi \int_{0}^{1} D_{t, j} d X_{t, j}^{(i)}-(1-r \phi) \int_{0}^{1} d X_{t, j}^{(i)}\right] \\
& -\left(F_{t+1}-F_{t}\right) .
\end{aligned}
$$

Next use the fact $\bar{D}_{t+1, j}=\rho \bar{D}_{t, j}+(1-\rho) \bar{\varepsilon}_{t+1, j}$ along with $\phi=\frac{\rho}{1+r-\rho},(1+\phi) \rho=(1+r) \phi$, and $(1+\phi)(1-\rho)=(1-r \phi)$ inside $(71)$ to arrive at

$$
c_{t+1, i}-c_{t, i}=\left(\frac{r}{1+r-\rho}\right)(1-\rho) \int_{0}^{1}\left(\varepsilon_{t+1, j}-1\right) d X_{t, j}^{(i)}-\left(F_{t+1}-F_{t}\right) .
$$

Having established (72), the dynamics of agent $i$ 's consumption under our postulate, we next 
turn attention to the Euler equations, starting with the bond Euler equation

$$
1=\beta(1+r) E_{t} e^{-\gamma\left(c_{t+1, i}-c_{t, i}\right)} .
$$

Substituting (72) into (73) and noting that $\int_{0}^{1}\left(\varepsilon_{t+1, j}-1\right) d X_{t, j}^{(i)}$ is normally distributed with mean zero and variance $\omega\left(\Delta_{t}\right)$ gives

$$
1=\beta(1+r) e^{\frac{\gamma^{2}}{2}\left(\frac{r}{1+r-\rho}\right)^{2}(1-\rho)^{2} \omega\left(\Delta_{t}\right)-\gamma F_{t}} E_{t}\left(e^{\gamma F_{t+1}}\right) .
$$

Now suppose that for any $r$ and a given desired distribution $\Psi(\Delta)$ we set

$$
F_{t}\left(\Delta_{t} ; r\right)=\frac{M}{\gamma}+\frac{\gamma}{2}\left(\frac{r}{1+r-\rho}\right)^{2}(1-\rho)^{2} \omega\left(\Delta_{t}\right)
$$

Then equation (74) can be written as (30). Since $(1+r) E e^{\frac{\gamma^{2}}{2}\left(\frac{r}{1+r-\rho}\right)^{2}(1-\rho)^{2} \omega(\Delta)}$ is equal to 1 when $r=0$ and increases monotonically to infinity as $r$ increases, it follows that there exists a unique positive $r$ such that equation (30) holds. For that value of $r$, all investors' bond Euler equations are satisfied.

Finally, we need to determine $\Phi_{t}$ so as to ensure that the Euler equations for risky assets hold, i.e., that

$$
P_{t, j}=\beta E_{t}\left[e^{-\gamma\left(c_{t+1, i}-c_{t, i}\right)}\left(P_{t+1, j}+D_{t+1, j}\right)\right] .
$$

To that end, we use (31) and (28) to express (76) as

$$
\frac{1}{r}-\Phi_{t}+\phi\left(D_{t, j}-1\right)=\beta E_{t}\left[e^{-\gamma\left(c_{t+1, i}-c_{t, i}\right)}\left(\frac{1}{r}-\Phi_{t+1}+(1+\phi)\left(\rho D_{t, j}+(1-\rho) \varepsilon_{t+1, j}\right)-\phi\right)\right] .
$$

We next note that

$$
\beta E_{t}\left[e^{-\gamma\left(c_{t+1, i}-c_{t, i}\right)}\right](1+\phi) \rho D_{t, j}=\frac{(1+\phi) \rho}{1+r} D_{t, j}=\phi D_{t, j}
$$

using (73). Equation (78) simplifies (77) to

$$
\begin{aligned}
\frac{1}{r}-\Phi_{t}-\phi= & \beta E_{t}\left[e^{-\gamma\left(c_{t+1, i}-c_{t, i}\right)}\left(\frac{1}{r}-\Phi_{t+1}+(1+\phi)(1-\rho) \varepsilon_{t+1, j}-\phi\right)\right] \\
= & \frac{1}{r(1+r)}-\beta E_{t}\left[e^{-\gamma\left(c_{t+1, i}-c_{t, i}\right)} \Phi_{t+1}\right]+\frac{1}{1+r}(-\phi+(1+\phi)(1-\rho)) \\
& +(1+\phi)(1-\rho) \beta E_{t}\left[e^{-\gamma\left(c_{t+1, i}-c_{t, i}\right)}\left(\varepsilon_{t+1, j}-1\right)\right] .
\end{aligned}
$$

Using (72), Stein's Lemma, the fact that $\operatorname{cov}\left(\int_{0}^{1}\left(\varepsilon_{t+1, j}-1\right) d X_{t, j}^{(i)}, \varepsilon_{t+1, j}\right)=\omega(\Delta)$ (see Proposition 1), and (73) implies

$$
\beta E_{t}\left[e^{-\gamma\left(c_{t+1, i}-c_{t, i}\right)} \varepsilon_{t+1, j}\right]=\frac{1-\gamma \frac{r}{1+r-\rho}(1-\rho) \omega\left(\Delta_{t}\right)}{1+r} .
$$


Substituting (80) into (79) gives linear equations in $\Phi_{0}$ and $\Phi_{1}$, solved by (32), respectively (33).

To complete the proof of the claim that $\Delta_{t}$ is chosen optimally, we provide an explicit example of a family of functions for $F_{t}(\Delta)$ that has the desired properties. To start, we compute the value function of an investor adopting the policies of Proposition 5. Equation (73) along with (72) imply that

$$
\begin{aligned}
V\left(W_{t, i}, \Delta_{t}\right) & =-\frac{1}{\gamma} \sum_{t \geq 0} \beta^{t} E_{t}\left[e^{-\gamma c_{t, i}}\right]=-\frac{1}{\gamma} e^{-\gamma c_{0, i}}\left(1+\sum_{t \geq 1} \beta^{t} E_{t}\left[e^{-\gamma \sum_{m=0}^{t-1}\left(c_{m+1, i}-c_{m, i}\right)}\right]\right) \\
& =-\frac{1}{\gamma} e^{-\gamma c_{0, i}} \sum_{t \geq 0}(1+r)^{-t}=-\frac{1+r}{\gamma r} e^{-\gamma c_{0, i}} .
\end{aligned}
$$

In turn, equations (66), (68), and (75) imply

$$
V\left(W_{t, i}, \Delta_{t}\right)=-\frac{1+r}{\gamma r} e^{-\frac{\gamma r}{1+r} W_{t, i}+z\left(\Delta_{t}\right)},
$$

where $z_{t}\left(\Delta_{t}\right) \equiv-\frac{r}{1+r} \gamma \Phi_{t}+M+\frac{\gamma^{2}}{2}\left(\frac{r}{1+r-\rho}\right)^{2}(1-\rho)^{2} \omega\left(\Delta_{t}\right)$.

Next we suppose that we no longer impose that the investor choose $\Delta=\Delta_{t}$, (where $\Delta_{t}$ is the time- $t$ random draw of $\Delta$ that we imposed in Proposition 5 ). Instead $\Delta$ is chosen optimally. However, prices are still given by $P_{t, j}\left(\Delta_{t}, D_{t, j}\right)$ from equation (31). We will construct a function $\kappa_{t} g_{t}(\Delta)$ that renders the choice $\Delta=\Delta_{t}$ optimal at the total cost specified in (75).

Throughout we let $\bar{X}_{t}^{(i)}\left(\Delta ; \Delta_{t}\right)$ denote the optimal number of total risky assets chosen by investor $i$, and assuming that that investor chooses $\Delta$ and prices are given by $P_{t, j}\left(\Delta_{t}, D_{t, j}\right)$. For future reference, we note that by construction of the price function $P_{t, j}\left(\Delta_{t}, D_{t, j}\right)$ it follows that $\bar{X}_{t}^{(i)}\left(\Delta_{t} ; \Delta_{t}\right)=1$. Using (81) the first order condition characterizing an optimal $\Delta$ is

$$
F_{t}^{\prime}(\Delta)=h\left(\Delta ; \Delta_{t}\right)
$$

where

$$
h\left(\Delta ; \Delta_{t}\right)=-\frac{1}{1+r} \frac{\gamma}{2}\left(\frac{r}{1+r-\rho}\right)^{2}(1-\rho)^{2}\left(\bar{X}_{t}^{(i)}\left(\Delta ; \Delta_{t}\right)\right)^{2} \omega^{\prime}(\Delta) .
$$

Next we fix a value of $\Delta_{t}$ and we simplify notation by writing $h(\Delta)$ rather than $h\left(\Delta ; \Delta_{t}\right)$. We also let $q(x)$ denote some continuous function with $q(0)=1$ and $q(x)>1$ for $x>0$. Let $\eta \in[0,1]$, take some positive (small) $\varepsilon<\frac{\Delta_{t}}{2}$, and consider the function

$$
F_{t}^{\prime}(\Delta)=\left\{\begin{array}{ll}
\frac{\Delta}{\varepsilon} \eta h(\varepsilon) & \text { for } \Delta \leq \varepsilon \\
\eta h(\Delta) & \text { for } \Delta \in\left(\varepsilon, \Delta_{t}-\varepsilon\right] \\
\eta h\left(\Delta_{t}-\varepsilon\right) \frac{\Delta_{t}-\Delta}{\varepsilon}+h\left(\Delta_{t}\right) \frac{\Delta-\Delta_{t}+\varepsilon}{\varepsilon} & \text { for } \Delta \in\left(\Delta_{t}-\varepsilon, \Delta_{t}\right] \\
h(\Delta) q(\Delta) & \text { for } \Delta>\Delta_{t}+\varepsilon .
\end{array} .\right.
$$

By construction, $F_{t}^{\prime}(0)=0$ and $F_{t}^{\prime}(\Delta)$ is continuous and increasing in $\Delta$. More importantly, $F_{t}^{\prime}\left(\Delta_{t}\right)=h\left(\Delta_{t}\right)$, and hence $\Delta=\Delta_{t}$ satisfies the necessary first order condition (82). Moreover, since $F_{t}^{\prime}(\Delta)<(>) h(\Delta)$ for $\Delta<(>) \Delta_{t}$, it follows that $\Delta=\Delta_{t}$ is optimal for any $\varepsilon>0$ and 
$\eta \in[0,1]$. Finally,

$$
\lim _{\varepsilon \rightarrow 0} \int_{0}^{\Delta_{t}} F_{t}^{\prime}(\Delta)=\eta \int_{0}^{\Delta_{t}} h(x) d x>0 .
$$

Now suppose that nature draws $\Delta_{t}=\Delta_{u}>0$. By choosing $M$ that is sufficiently close to $-\frac{\gamma}{2}\left(\frac{r}{1+r-\rho}\right)^{2}(1-\rho)^{2} \omega(\bar{\Delta})$ it follows that

$$
0<\frac{M}{\gamma}+\frac{\gamma}{2}\left(\frac{r}{1+r-\rho}\right)^{2}(1-\rho)^{2} \omega\left(\Delta_{u}\right)<\int_{0}^{\Delta_{u}} h(x) d x
$$

Combining equations (84) and (85) it follows that for sufficiently small $\varepsilon>0$ there exists some $\eta \in[0,1]$ so that

$$
\int_{0}^{\Delta_{u}} F_{t}^{\prime}(x) d x=\frac{M}{\gamma}+\frac{\gamma}{2}\left(\frac{r}{1+r-\rho}\right)^{2}(1-\rho)^{2} \omega\left(\Delta_{u}\right)>0 .
$$

Hence, when $\Delta_{t}=\Delta_{u}$ the cost function $\kappa_{t} g_{t}(\Delta)$ renders $\Delta=\Delta_{u}$, while also satisfying (75). The same argument implies that for any value of $\Delta_{t}$ that satisfies

$$
0<\frac{M}{\gamma}+\frac{\gamma}{2}\left(\frac{r}{1+r-\rho}\right)^{2}(1-\rho)^{2} \omega\left(\Delta_{t}\right)<\int_{0}^{\Delta_{t}} h(x) d x,
$$

there exists $\eta \in[0,1]$ and sufficiently small $\varepsilon>0$ such that the optimal $\Delta$ coincides with $\Delta_{t}$, and

(75) holds. Continuity of $\omega\left(\Delta_{t}\right)$ and of $\int_{0}^{\Delta_{t}} h(x) d x$ in $\Delta_{t}$ implies that as long as $\underline{\Delta}$ is sufficiently close to $\Delta_{u}$, there always exists $\eta \in[0,1]$ and $\varepsilon>0$ (both depending on the random draw $\Delta_{t}$ ) such that $\Delta=\Delta_{t}$ is optimal and (75) holds.

Proof of Proposition 6. Parts (i)-(iii) are proved in the main body of the text. Part (iv) comes down to noticing that

$$
\operatorname{cov}\left(e^{z}, z\right)>0
$$

for any random variable $z$ - in particular, for $z=\omega(\Delta)$. The second statement of (iv) follows from the first and Jensen's inequality applied to the convex function $\omega$.

\section{An interpretation of participation costs}

Throughout the paper we maintain the assumption that participation in "distant" markets incurs participation costs. In this appendix we discuss how these costs could arise as informationacquisition costs that permit an investor to avoid the lower net returns earned by an investor unfamiliar with the asset class. Indeed, we wish to re-emphasize that we do construe the notion of distance broadly, as a stand-in for the level of familiarity of investors in one location with all aspects of the financial environment in another.

We start by summarizing the set-up in Gârleanu et al. (2013), on which the interpretation is based. While we could simply refer the reader to this paper, we take this route in the interest of staying self-contained and keeping the effort required of the interested reader to a minimum. 
Just as in the present model, investors are aligned on a circle. In each location of the circle, investors are of two types: a) "common" investors, who are endowed with local "regular" stocks that pay an exogenous, location-specific dividend, with the same properties as in the present paper; and b) "inside" investors, endowed with "fraudulent" firms in that location. Fraudulent firms produce lower dividends than regular firms - for simplicity it is assumed that they produce a dividend of zero. However, if insiders choose to, they can manipulate the earnings of their firms - for instance by diverting their personal funds into the company to boost the firm's dividends. This assumption ensures that the insider always makes a trading profit by trading in her firm, irrespective of whether she buys or sells stock in her firm. ${ }^{29}$

Similar to the present paper, investors see an incentive to invest in distant locations for diversification reasons. However, when investing in a location other than her own, an incompletely informed investor does not know which stocks are regular and which are fraudulent. Investors receive a signal about the type of every firm in every location. The quality of this signal deteriorates with the distance separating the investor from the firm. Hence, even though diversification benefits increase with distance, so does the informational disadvantage.

Gârleanu et al. (2013) shows that there exists an equilibrium in which insiders strategically manipulate the prices of the stocks they are endowed with, so that the prices of all risky securities in a given location are equal. The ability of insiders to manipulate the earnings of their firms implies that no trade with such an insider can be profitable for any other investor. This deters local short sellers from shorting the stock of the fraudulent firms. ${ }^{30}$ Since prices are the same for fraudulent and regular securities, investors use only their private information in weeding out potential insiders and avoiding trading with them.

Because the ability to distinguish fraudulent from regular securities declines with distance, investors prefer to invest close to their own location and forego diversification opportunities. Indeed, in a special case where investors receive a (perfectly informative) signal for a subset of locations, and an uninformative signal for the rest, they choose to invest only in locations where they are informed, similar to the present paper.

Gârleanu et al. (2013) assumes that investors are simply endowed with their signals and doesn't consider the possibility of incurring costs to expand the set of informative signals (and by implication the participation interval on which the investor chooses to invest.) By contrast, the focus of the present paper is to study investors' incentives to to expand, by paying appropriate costs, the set of locations in which they can participate. Indeed, the endogeneity of the participation decision is at the core of many results in this paper, since it is responsible for the emergence of non-convexities, the diversity of financial strategies, the emergence of endogenous leverage, the vulnerability of the market equilibrium to sudden reversals in participation, etc.

\section{An alternative formulation of the leverage constraint}

In this section we elaborate further on the interaction between borrowing constraints and high price sensitivity to participation costs. Specifically, we introduce a "limited-liability" constraint that

\footnotetext{
${ }^{29}$ Vila (1989) shows the possibility of profitable manipulation, when the payoffs of a security can be manipulated.

${ }^{30}$ The model assumes that local investors only observe prices, and not the demands expressed by "distant" investors for local fraudulent firms. Hence, if they sold short they would not know whether they were trading against less informed investors or the insider.
} 
places an endogenous bound on borrowing, and show that it plays a similar role to constraint (23) in the text. In particular, we recover the conclusions of Section 5.

Before formalizing and analyzing the constraint, we provide a new dividend structure. An important novel feature of this structure is that all dividends are non-negative, so that the notion of limited liability is economically meaningful. Specifically, let $\Gamma_{j}$ be a Gamma process on $[0,1)$, so that for $u>s$ we have

$$
\Gamma_{u}-\Gamma_{s} \sim \Gamma(k(u-s) ; \nu) .
$$

Extending $d \Gamma$ to the entire real line as before — that is, via $d \Gamma_{s}=d \Gamma_{s \bmod 1}$ — we define

$$
D_{j}=\mu+\int_{j-\frac{1}{2}}^{j+\frac{1}{2}} w_{s-j} d \Gamma_{s}
$$

for some $\mu \geq 0$ and weights $w_{i} \geq 0$ periodic with period 1 and symmetric around 0 . In the interest of concreteness, in our numerical illustration below we define $w_{i}=1$ if $i \in\left[-\frac{1}{4}, \frac{1}{4}\right]$ and $w_{i}=0$ otherwise. Conveniently, for this choice of $w, D_{j}$ and $D_{j+\frac{1}{2}}$ are independent.

More important, specification (89) generally implies that dividends are positive and the joint distribution of the dividends in any $n$ locations depends exclusively on the distances on the circle between the locations.

An agent located in location $i$ maximizes utility over end-of-period wealth $W_{1, i}$ net of participation costs, that is, she maximizes

$$
-\frac{1}{\gamma} E_{0} e^{-\gamma\left(W_{1, i}-F_{i}\right)}
$$

where $F_{i}$ refers to the participation costs incurred by the agent, depending on her participation choices. For the participation costs we adopt the same structure as in Section 5. Specifically we assume that by paying a cost $\kappa$, an investor can participate not only in her location but also in the location diametrically "opposite" hers on the circle. Otherwise the investor can only invest in the risky asset in her own location. Proceeding as in Section 5, the indifference of agent $i$ between investing exclusively in location $i$ and incurring the cost $\kappa$ to participate also in location $i+\frac{1}{2}$ means

$$
\max _{1-w_{2}^{f}}-E e^{-\gamma\left(\frac{1}{2}\left(1-w_{2}^{f}\right) \sum_{j=i, i+\frac{1}{2}}\left(D_{j}-P\right)-\kappa\right)}=\max _{1-w_{1}^{f}}-E e^{-\gamma\left(\left(1-w_{1}^{f}\right)\left(D_{i}-P\right)\right)},
$$

where we have used the definition of an agent's objective and her budget constraint. Note that $1-w_{2}^{f}$, respectively $1-w_{1}^{f}$, is the leverage choice of an agent who decides to invest across the two locations, respectively only in her own location.

In the absence of any constraint on leverage, the implicit function theorem applied to equation (90) yields

$$
\frac{d P}{d \kappa}=-\frac{1}{w_{1}^{f}-w_{2}^{f}}<0,
$$

exactly as in Section $5 .^{31}$

Now suppose that, due to the no-recourse nature of lending contracts, borrowing is restricted so

\footnotetext{
${ }^{31}$ It is possible to show that there exist values of $\kappa$ for which only asymmetric equilibria exist.
} 

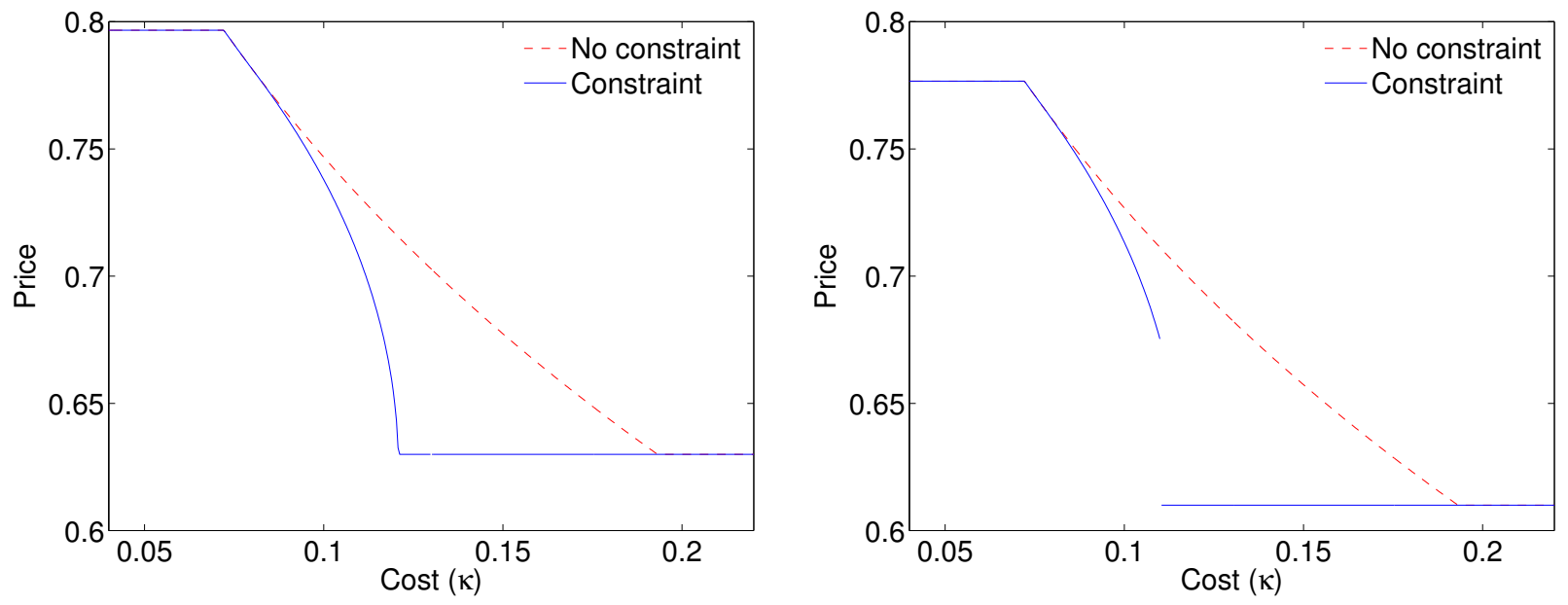

Figure 13: The figure illustrates the higher sensitivity of the price to the diversification cost in the presence of the constraint. In the left panel, the price decreases continuously to the value obtaining with no diversification. In the right panel, the price jumps to this value when $\kappa=\mu$. In either case, the slope of the solid line (the price in the presence of a leverage constraint) exhibits a steeper decline then the dotted line (the price in the absence of a leverage constraint). The common parameters used here are $k=20, v=10$, and $\gamma=10$; in the left panel $\mu=0.13$, while in the right one $\mu=0.11$.

as to ensure that there is no default in equilibrium. ${ }^{32}$ Thus, borrowing is subject to the constraint

$$
X_{2}^{S} D^{\min }+\left(X_{2}^{B}-\kappa\right) \geq 0
$$

where $D^{\text {min }}$ is the smallest possible dividend in period $1, X_{2}^{S}$ is the number of shares chosen by investor $a$, and $-X_{2}^{B}$ is the amount borrowed by the investor. Using the time-zero budget constraint and noting that $D^{\min }=\mu$ and $X_{2}^{S}-1=-w_{2}^{f}$, equation (92) becomes

$$
-w_{2}^{f}(P-\mu) \leq \mu-\kappa .
$$

Equation (93) is key to the assertion that modeling the constraint in the way we do here induces small changes in $\kappa$ to translate into larger drops in the price than would obtain absent the constraint. Repeating the steps in Section 5 we get

$$
\frac{d P}{d \kappa}=-\frac{1+\lambda}{w_{2}^{f}-(1+\lambda) w_{1}^{f}}<-\frac{1}{w_{2}^{f}-w_{1}^{f}}<0 .
$$

The intuition for the increased price sensitivity, captured by (94), is quite immediate: An increase in $\kappa$ not only requires the price to decrease in order to avoid a decrease in $V_{2}$ relative to $V_{1}$ (the effect behind equation (91)); it also pushes the price down to counteract the direct effect

\footnotetext{
${ }^{32}$ Richer contracts, through which the borrower and lender share some risk, can be envisaged. Note, however, that such a contract would allow an agent (partial) diversification across locations at zero cost, and thus run counter to the central friction of the paper.
} 
of tightening of the constraint (decreasing the right-hand-side of (93)).

Depending on the parameters, the price may even drop discontinuously to the value obtaining in the no-diversification equilibrium. The point is made most starkly in the case $\kappa=0$ and $\mu=0$. At these values there is diversification, and no leverage. Any increase in $\kappa$, on the other hand, drives the price discontinuously down to the no-diversification value. More generally, it can happen that, as $\kappa$ approaches $\mu$, enough agents continue to diversify — even if their leverage is virtually zero - for the price to be above the no-diversification value that obtains when $\kappa>\mu$. However, once $\kappa$ exceeds $\mu$ the price drops discontinuously, as the right panel of Figure 13 illustrates.

To conclude, even if one modeled borrowing limitations as resulting from a no-default requirement, the price function is steeper in $\kappa$ than when the constraint is absent, and can even be discontinuous. 